\title{
Rubber rolling over a sphere
}

\author{
Jair Koiller* \\ Fundação Getulio Vargas \\ Praia de Botafogo 190 \\ Rio de Janeiro, RJ, 22250-040, Brazil (jkoiller@fgv.br) \\ Kurt Ehlers \\ Truckee Meadows Community College \\ 7000 Dandino Boulevard \\ Reno, NV, 89512-3999, USA (kehlers@tmcc.edu)
}

August 6, 2018

Key words: Nonholonomic mechanics, Reduction, Chaplygin systems

AMS MSC(2000); 37J60, 70F25, 58A15, 58A30 .

\begin{abstract}
Rubber" coated rolling bodies satisfy a no-twist in addition to the no slip satisfied by "marble" coated bodies [28]. Rubber rolling has an interesting differential geometric appeal because the geodesic curvatures of the curves on the surfaces at the corresponding points are equal. The associated distribution in the 5 dimensional configuration space has 2-3-5 growth (these distributions were first studied by Cartan; he showed that the maximal symmetries occurs for rubber rolling of spheres with 3:1 diameters ratio and materialize the exceptional group $G_{2}$ ). The 2-3-5 nonholonomic geometries are classified in a companion paper [29] via Cartan's equivalence method [19]. Rubber rolling of a convex body over a sphere defines a generalized Chaplygin system [21, 57, 35, 27, 39] with $S O(3)$ symmetry group, total space $Q=S O(3) \times S^{2}$ and base $S^{2}$, that can be reduced to an almost Hamiltonian system in $T^{*} S^{2}$ with a non-closed 2-form $\omega_{N H}$. In this paper we present some basic results on the spheresphere problem: a dynamically asymmetric but balanced sphere of radius $b$ (unequal moments of inertia $I_{j}$ but with center of gravity at the geometric center), rubber rolling over another sphere of radius $a$. In this example $\omega_{N H}$ is conformally symplectic [59]: the reduced system becomes Hamiltonian after a coordinate dependent change of time. In particular there is an invariant measure, whose density is the determinant of the reduced Legendre transform, to the power $p=\frac{1}{2}\left(\frac{b}{a}-1\right)$. Using sphero-conical coordinates we verify the results by Borisov and Mamaev [12 13] that the system is integrable for $p=-1 / 2$ (ball over a plane) and $p=-3 / 2$ (rolling ball with twice the radius of a fixed internal ball).
\end{abstract}

\footnotetext{
${ }^{*}$ On a CAPES-Fulbright visit to Caltech, Winter 2005-2006.
} 


\section{Contents}

1 Introduction 3

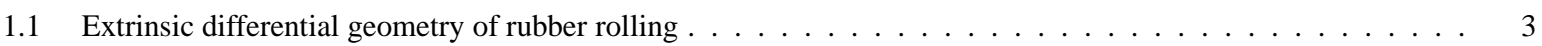

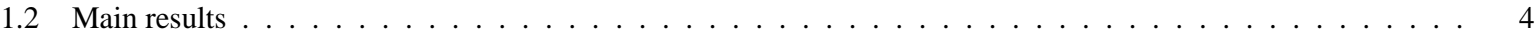

2 Preliminaries 6

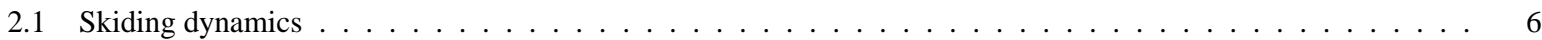

2.2 No slip and no-twist constraints: kinematical relations for the Poisson vector . . . . . . . . . . . . . . . 7

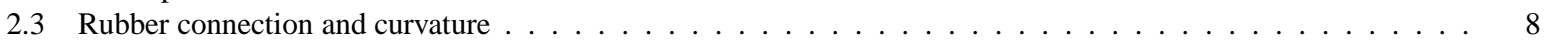

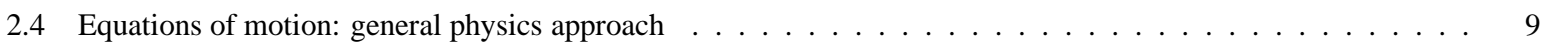

2.5 Marble Chaplygin sphere over a sphere or a plane . . . . . . . . . . . . . . . . . . . . 10

3 Rubber rolling over a sphere as a $S O(3)$ Chaplygin system.

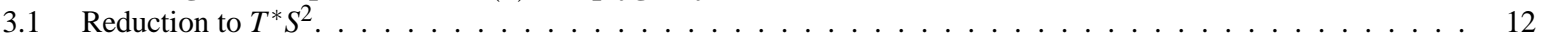

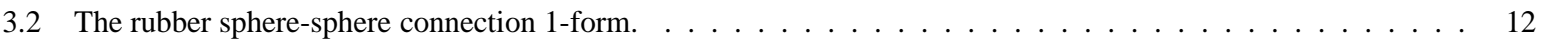

3.3 Curvature of the sphere-sphere connection . . . . . . . . . . . . . . . . . . . . . . . . . . 14

3.4 Hamiltonization of the sphere-sphere system $\ldots \ldots \ldots \ldots \ldots$

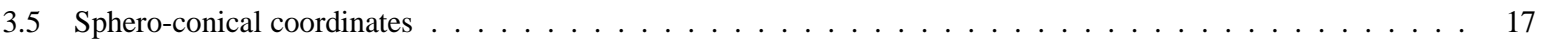

4 Some directions for research 19

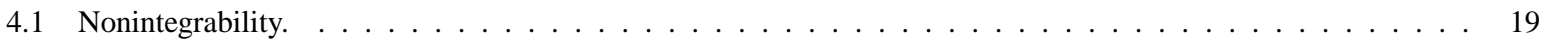

4.2 Rolling with sliping but no twisting and Rattleback phenomena . . . . . . . . . . . . . . . . . . . . 19

4.3 Skiding dynamics: mathematical hockey . . . . . . . . . . . . . . . . . . . . . . . . 19

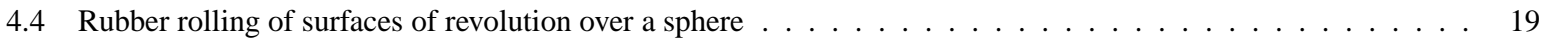

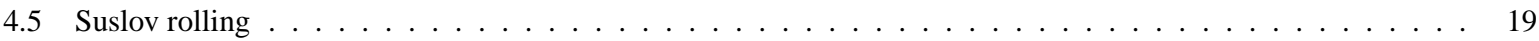

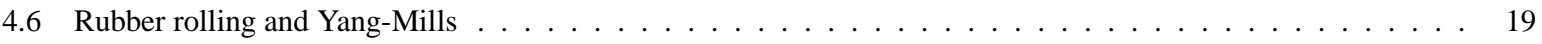




\section{Introduction}

Given a riemannian manifold $Q^{n}$ and a $s<n$ dimensional distribution $\mathcal{D}$ of subspaces of $T Q$, two different theories apply. One is subriemannian geometry [51] which appears in the study of underactuated control systems and gauge theories. Nonholonomic geometry [2, 24, 53, 5, 25] follows d'Alembert's principle, and describes mechanical systems with nonintegrable constraints 1 .

This an a companion [29] paper belong to nonholonomic geometry. We use the following terminology: "Rubber" coated bodies means adding the no-twist condition to the usual no-slip constraints of "marble" coated bodies. The dynamics for the former lives on a 7 dimensional phase space, 8 dimensional for the latter. "Waxed" marble bodies have no velocity constraints: skiding dynamics lives on a 10 dimensional phase space.

Strangelly enough, while marble bodies have been extensively studied in the NH-literature, rubber rolling seems to have been neglected. This is curious because they should be easier to study and furthermore, rubber rolling has an appealing differential geometric interpretation.

In this paper we provide details of some results on rubber rolling over a sphere announced in [28]. Details about the classification of 2-3-5 nonholonomic geometries via Cartan's equivalence [19] will be submitted elsewhere. The expert can go directly to section 3, or pass quickly over section 2. Although we tried to be reasonably self-contained, we use freely the geometric mechanics jargon, see eg. [1, 2, 49, 54]. We also presented an extensive but focused list of references 2 .

Using sphero-conical coordinates we verify the results of Borisov and Mamaev [12, 13] that the system is integrable for $b / a=0$ and $b / a=-2$. A table of results similar to that of Borisov and Mamaev (tables 1 and 2 of [10]) for marble bodies rolling over a plane or a sphere) is in order.

\subsection{Extrinsic differential geometry of rubber rolling}

In this section we discuss the implications of rolling a surface $\Sigma_{2}$ without sliping or twisting over a surface $\Sigma_{1}$ from the extrinsic differential geometric perspective. In [29] we show that this process belongs actually to their intrinsic geometries.

Given a curve $C$ in a surface $\Sigma$, define the $(C, \Sigma)$ adapted frame as the moving frame $F=(t, u, N)$, where $t$ is the tangent vector, $N$ the normal to the surface (so an orientation is chosen) and $u=N \times t$ is the surface normal to the curve. Denoting ${ }^{\prime}=d / d s$ the derivative with respect to arc length, as it is well known we have

$$
t^{\prime}=\kappa_{g} u+\kappa_{n} N, u^{\prime}=-\kappa_{g} t-\tau_{g} N, N^{\prime}=-\kappa_{n} t+\tau_{g} u
$$

where $\kappa_{g}=t^{\prime} \cdot u$ is called the geodesic curvature, $\kappa_{n}=t^{\prime} \cdot N$ is the normal curvature, and $\tau_{g}=N^{\prime} \cdot u$ the geodesic torsion. Recall an elementary result from classical differential geometry

Proposition 1 (see e.g. Struik, [58], p.201, exercise 2, section 4-8.) The geodesic curvature of a curve C on a surface $S$ is equal to the ordinary curvature of the plane curve into which $C$ is deformed when the developable surface enveloped by the tangent planes to $S$ along $C$ is rolled out on a plane.

Remark 1 The inverse problem is hard: finding a curve with prescribed geodesic curvature on a surface $S$ with metric ds ${ }^{2}=$ $E d u^{2}+2 F d u d v+G d v^{2}$ gives rise to a nonlinear second order equation for $u(s), v(s)$.

Writing $F$ as the orthonormal matrix with colums t,u,N, then $F^{-1} F^{\prime}=A$, where $A$ is the skew symmetric matrix

$$
A=\left(\begin{array}{lll}
0 & -\kappa_{g} & -\kappa_{n} \\
\kappa_{g} & 0 & \tau_{g} \\
\kappa_{n} & -\tau_{g} & 0
\end{array}\right) .
$$

Let us write the structure equations 1.1 as

$$
t^{\prime}=\omega_{F} \times t, u^{\prime}=\omega_{F} \times u, N^{\prime}=\omega_{F} \times N, \omega_{F}=-\tau_{g} t-\kappa_{n} u+\kappa_{g} N .
$$

This yiels a nice dynamic interpretation: as the curve is traversed with unit velocity, the frame is rotating instantaneously around the vector $\omega_{F}$ with angular velocity $\left\|\omega_{F}\right\|$.

Consider two surfaces $\Sigma_{2}$ and $\Sigma_{1}$ an suppose that $\Sigma_{2}$ moves always "touching" $\Sigma_{1}$. We neglect physical intersections that may occur if one of them is not convex). At the contact point the normals will be equal or opposite (depending on which orientations one choses). We use the following conventions: for a closed convex $\Sigma_{2}$, the exterior normal defines its orientation; if $\Sigma_{1}$ is also convex we take the opposite orientations when they roll externally to each other.

\footnotetext{
${ }^{1}$ Hertz [34] introduced the word nonholonomic to designate nonintegrable distributions and was the first to call attention that, although using the same ingredients, the two theories are quite different.

${ }^{2}$ We apologize for omissions, specially on nonholonomic reduction and special geometric structures (like almost Poisson and almost Dirac); we just mention some new connections of nonholonomic systems in robotics and control [55], 62].
} 
When there is no sliding, the tangent vectors at the corresponding curves of points of contact have the same length, so we may use the arc length as a common parameter for both curves. Let $C_{1}: x=x_{1}(s)$ and $C_{2}: x=x_{2}(s)$ denote the corresponding curves of points of contact in $\Sigma_{1}$ and $\Sigma_{2}$. and let us describe the rolling action

$$
g(s)=(R(s), a(s)) \in S E(3)
$$

( $g$ acts on $x \in \mathbf{R}^{3}$ by $g \cdot x=R x+a$, meaning first rotate, then translate").

Let $\omega_{R}$ the angular velocity in space of the rotation, and assume the condition $\omega_{R} \times x_{2}+R^{-1} \dot{a}=0$. Then $R \dot{x}_{2}=\dot{x}_{1}$ and in fact the corresponding adapted frames are related by

$$
R(s) F_{2}(s)=F_{1}(s)
$$

i.e, they match under the action of $R(s)$. Here we orient $\Sigma_{1}$ so that the normals to the surfaces point in the same direction. Differentiating, we get

$$
F_{2}^{-1}\left(R^{-1} R^{\prime}\right) F_{2}+F_{2}^{-1} F_{2}^{\prime}=F_{1}^{-1} F_{1}^{\prime}
$$

There is no loss in generality in assuming that at the point of contact corresponding to $s=0$ we have $F_{1}(0)=F_{2}(0)=i d$ (so $R(0)=i d$, and $x_{1}(0)=x_{2}(0)=0$. Therefore, if $R^{\prime}(0)$ is the skew-symmetric matrix $B$ given by

$$
B_{12}=-\omega_{3}, b_{13}=\omega_{2}, a_{23}=-\omega_{1},
$$

then

$$
\omega_{F_{1}}=\omega_{F_{2}}+\omega_{R}
$$

which gives the following relation 3 between the invariants of the curves $C_{1}$ and $C_{2}$ :

$$
\kappa_{g}^{(1)}=\kappa_{g}^{(2)}+\omega_{3}, \kappa_{n}^{(1)}=\kappa_{n}^{(2)}-\omega_{2}, \tau_{g}^{(1)}=\tau_{g}^{(2)}-\omega_{1}
$$

A very important consequence for our purposes is the following

Proposition 2 Under the condition of no twisting $\left(\omega_{3}=0\right)$ the geodesic curvatures of the contact curves at the corresponding points are the same.

\subsection{Main results}

Rubber rolling of a convex body $\mathcal{B}$ over a sphere of radius a yields a generalized Chaplygin system, see [27, 31, 39, 57]. We have a principal bundle with total space $Q=S O(3) \times S^{2}$ and base space $B=S^{2}$. The symmetry group $G$ is $S O(3)$, acting diagonally. The metric is left invariant, and the constraints define a connection on the principal bundle, called the "rubber connection".

The theory developed in [27] implies that the dynamics reduces to $T^{*} S^{2}$, with a non-closed 2-form $\omega_{n h}=\omega_{\text {can }}^{T^{*} S^{2}}+(J, K)$ and a "compressed" Hamiltonian. The term $(J, K)$ is a semi-basic form, where $J$ is the momentum map and $K$ is the curvature of the connection. The configuration variable in the base, $\gamma \in S^{2}$, has a nice geometric interpretation. It is the Poisson vector, namely, minus the external normal vector of the rolling body at the contact point (or equivalently, the normal vector to the base sphere or plane), seen in the body frame.

If a solution $\gamma(t)$ is found, then the angular velocity $\Omega(t)$ of $\mathcal{B}$ in the body frame is the horizontal lift of $\dot{\gamma}$ via the rubber connection. To reconstruct the attitude matrix one needs to solve a linear system with time dependent coefficients, $\dot{R}=R[\Omega(t)]$. To complete the reconstruction, note that the contact point in $S_{a}$ is $q(t)=a R \gamma(t)$.

In principle, the curve $q(t)$ in $S_{a}$ could also be reconstructed from $Q(t)=d N_{\mathcal{B}}^{-1}\left(-\gamma(t)\right.$ ) on $S_{b}$ (where $d N_{\mathcal{B}}$ is the Gauss map of $\mathcal{B}$ ) using the fact that the geodesics curvatures $\kappa_{g}$ of $q(t)$ and $Q(t)$ at corresponding points are the same. If one succeeds to solve this nonlinear equation, then the rotation matrix $R$ can be computed, by noting that it takes the adapted frame $(\dot{Q} /|\dot{Q}, \dot{Q} \times \gamma /| \dot{Q} \times \gamma \mid,-\gamma)$ along $Q(t)$ to the corresponding adapted frame $(\dot{q} /|\dot{q}, q / a \times \dot{q} /| \dot{q}, q / a)$, (modulo a trivial change of signs depending on the chosen orientations).

In passing, we observe that while it is simple to reconstruct a curve in the plane from its curvature $\kappa(s)$, for a general surface this task results on a complicated nonlinear equation (for $\kappa_{g}=0$ this is already the geodesic equations). For a spherical curve we get a linear system with variable coefficient $4 \frac{d^{2} q}{d s^{2}}=\frac{\kappa_{g}(s)}{a} q \times \frac{d q}{d s}-\frac{1}{a^{2}} q$.

In this paper we discuss the example where the surface of the rolling body $\mathcal{B}$ is a also a sphere, of radius $b$. It is a "Chaplygin sphere", meaning a sphere of mass $\mu$ where the center of mass is the geometric center, but dynamically asymmetric, i.e., unequal moments of inertia $I_{j}$. The metric is given by

$$
2 T=\mu(1 \pm b / a)^{2}\|\dot{q}\|^{2}+(A \Omega, \Omega), A=\operatorname{diag} I_{1}, I_{2}, I_{3}
$$

\footnotetext{
${ }^{3}$ We learned this result from Mark Levi [45].

${ }^{4}$ Is this solvable by quadratures?
} 
where $q \in S_{a}$ is the contact point and $\Omega, \omega$ are respectively the angular velocity of the rolling sphere $S_{b}$ with respect to body and space frames, respectively. The constraints are given by

$$
(1 \pm b / a) \dot{q}=( \pm b / a) \omega \times q, \dot{q} \cdot \omega=0 .
$$

(the plus sign corresponds to the external case).

Hence, when the rolling body is also a sphere, the constraints are invariant under the right action of $S O(3)$ on the second factor of $S^{2} \times S O(3)$. This system is (morally speaking) akin to a LR nonholonomic Chaplygin system [31]. For a true LR system with 2 dimensional base space one can guarantee the existence of a function $f: S^{2} \rightarrow \mathbf{R}$ such that $d\left(f \omega_{n h}\right)=0$. Moreover, the conformal factor $f$ would be $f=F^{-1 / 2}$, the inverse of the square root of the determinant of the reduced Legendre transform (identifying $T S^{2} \equiv T^{*} S^{2}$ ), where

$$
F(\gamma)=\operatorname{det}\left(\text { Leg }_{\text {red }}\right)=\left(I_{1} I_{2} I_{3}\right)\left(1+\frac{b}{a}\right)^{2}\left(\left(A^{-1} \gamma, \gamma\right)+\mu b^{2}\left[\frac{\gamma_{2}^{2}+\gamma_{3}^{2}}{I_{2} I_{3}}+\frac{\gamma_{1}^{2}+\gamma_{3}^{2}}{I_{1} I_{3}}+\frac{\gamma_{1}^{2}+\gamma_{2}^{2}}{I_{1} I_{2}}\right]+\frac{\mu^{2} b^{4}}{I_{1} I_{2} I_{3}}\right)
$$

Indeed, we will show that in the rubber sphere-sphere problem, the reduced system is Hamiltonizable. However, we get a different exponent:

$$
f_{a, b}(\gamma)=F^{\frac{b-a}{2 a}}, \gamma \in S^{2} .
$$

Probably an explanation for this mysterious exponent will come from a study of Chaplygin systems of the form $G \hookrightarrow$ $G \times H / G \rightarrow H / G$ where the base space is a homogeneous space, and $G$ acts diagonally in the total space. This theory should build up fom the LR systems $H \hookrightarrow G \rightarrow H / G$ studied by Fedorov and Jovanovic [31].

Borisov and Mamaev [12] observed that by taking suitable combinations of the reduced variables, solutions of rubber rolling of a sphere over a plane can be mapped to the solutions of Veselova's system [61, 60]. They have also shown integrability [13] (in this volume) in the case $b=-2 a$, where the fixed ball has half the radius and is internal to the rolling ball. We confirm their result by showing that the sphero-conical coordinates separates the Hamiltonian in the new time. Using sphero-conical coordinates $I_{1}<\lambda_{1}<I_{2}<\lambda_{2}<I_{3}$, given by

$$
\left(\gamma_{1}^{2}, \gamma_{2}^{2}, \gamma_{3}^{2}\right)=\left(\frac{\left(I_{1}-\lambda_{1}\right)\left(I_{1}-\lambda_{2}\right)}{\left(I_{1}-I_{3}\right)\left(I_{1}-I_{2}\right)}, \frac{\left(I_{2}-\lambda_{1}\right)\left(I_{2}-\lambda_{2}\right)}{\left(I_{2}-I_{3}\right)\left(I_{2}-I_{1}\right)}, \frac{\left(I_{3}-\lambda_{1}\right)\left(I_{3}-\lambda_{2}\right)}{\left(I_{3}-I_{1}\right)\left(I_{3}-I_{2}\right)}\right)
$$

Quantitatively, our main result can be given as follows:

Theorem 1 Let $(q, R) \in S_{a}^{2} \times S O(3)$ the configuration space coordinates, where $q$ is the contact point in the base sphere $S_{a}$ and $R$ the attitude matrix of the moving sphere $S_{b}$. Let $\gamma=R^{-1}(q / a)=-Q / b$ be the Poisson vector, where $Q$ is the contact point in the moving sphere, seen in the body frame. The equations of motion for $\gamma$ are governed, in a new time scale $\tau$ such that

$$
d \tau / d t=F(\gamma)^{\frac{b-a}{2 a}}, F(\gamma)=\left(1+\frac{b}{a}\right)^{2}\left(\lambda_{1}+\mu b^{2}\right)\left(\lambda_{2}+\mu b^{2}\right)
$$

by a Hamiltonian system (the nonholonomic vectorfield is $X_{n h}=F(\gamma)^{\frac{b-a}{2 a}} X_{H}$ )

$$
X_{H}: \frac{d \lambda}{d \tau}=H_{P}, \frac{d P}{d \tau}=-H_{\lambda}, 2 H=\frac{P_{1}^{2}}{\left[\left(\lambda_{1}+\mu b^{2}\right)\left(\lambda_{2}+\mu b^{2}\right)\right]^{\frac{b-a}{a}} c_{1}}+\frac{P_{2}^{2}}{\left[\left(\lambda_{1}+\mu b^{2}\right)\left(\lambda_{2}+\mu b^{2}\right)\right]^{\frac{b-a}{a}} c_{2}}
$$

where

$$
c_{1}=\frac{1}{4}\left(1+\frac{b}{a}\right)^{2}\left(\lambda_{2}-\lambda_{1}\right) \frac{\lambda_{2}+\mu b^{2}}{\left(\lambda_{1}-I_{1}\right)\left(I_{2}-\lambda_{1}\right)\left(I_{3}-\lambda_{1}\right)}, c_{2}=\frac{1}{4}\left(1+\frac{b}{a}\right)^{2}\left(\lambda_{2}-\lambda_{1}\right) \frac{\lambda_{1}+\mu b^{2}}{\left(\lambda_{2}-I_{1}\right)\left(\lambda_{2}-I_{2}\right)\left(I_{3}-\lambda_{2}\right)} .
$$

Corollary 1 The terms $\lambda_{2}+\mu b^{2}$ in $c_{1}$ and $\lambda_{1}+\mu b^{2}$ in $c_{2}$ are a nuisance for Hamilton-Jacobi separation, but they disappear in two cases, discovered first by Borisov and Mamaev [13]. One is seen immediately, $(b-a) / a=-1$, that is, $b / a \rightarrow 0$, the planar case. The other is $(b-a) / a=-3$, where we have the cross factors $\left(\lambda_{2}+m u b^{2}\right) /\left(\lambda_{1}-\lambda_{2}\right)$ in $P_{1}^{2}$ and $\left(\lambda_{1}+m u b^{2}\right) /\left(\lambda_{1}-\lambda_{2}\right)$ in $P_{2}^{2}$; to see that that the Hamiltonian also separates in this case, multiplying both sides of (1.12) by

$$
\frac{\lambda_{2}-\lambda_{1}}{\left(\lambda_{1}+m u b^{2}\right)\left(\lambda_{2}+m u b^{2}\right)}=\frac{1}{\lambda_{1}+m u b^{2}}-\frac{1}{\lambda_{2}+m u b^{2}}
$$

It is not known if there are hidden integrals for other values of $b / a$ and inertias $I_{j}$ (except for the case of two equal inertias), but this is unlikely. The case $b=a$ is special because the constraints are holonomic, so we have directly a two degrees of freedom Hamiltonian system. It was somewhat frustrating to realize that in this case the problem does not separate in sphero-conical coordinates and in fact seems to be chaotic from numerical experiment 5 .

${ }^{5}$ We admit losing a bet to Ivan Mamaev and Alexei Borisov, and we owe them a dinner in a barbecue house in Rio. 


\section{Preliminaries}

\subsection{Skiding dynamics}

As we all know, the boundary of a strictly convex body $\mathcal{B}$ is a closed surface $\Sigma_{2}$ with strictly positive Gaussian curvature at all points.

We say $\mathcal{B}$ is in standard position when 0 is the center of mass and the principal axis of inertia are aligned with $\mathrm{OX}, \mathrm{OY}, \mathrm{OZ}$ with moments of inertia $I_{1} \leq I_{2} \leq I_{3}$, respectively. Under a Euclidian motion $g=(x, R) \in S E(3)$ the surface goes to $g \cdot \Sigma_{2}$, the center of mass of $g \cdot \mathcal{B}$ is at $x \in \mathbf{R}^{3}$ and the three principal axis become, respectively, the columns $e_{1}, e_{2}, e_{3}$ of the attitude matrix $R$.

The Gauss mappings $N_{j}: \Sigma_{j} \rightarrow S^{2}$ are important ingredients for the sequel. We make no special requirement on $N_{1}$, but assuming $\Sigma_{2}$ to be convex is useful, it guarantees that $N_{2}$ is one to one and onto $S^{2}$.

The reader can easily sketch a figure to visualize our notation. Let $\Sigma_{1}$ be fixed and $\Sigma_{2}$ move under the action of $S E(3)$. The unconstrained configuration space is ten dimensional, $\left(S E(3) \times \Sigma_{2}\right) \times \Sigma_{1}$. When we impose the condition that the two bodies touch, the configuration space becomes five dimensional. One way to do the dimension count is to consider equations $F_{1}\left(x_{1}, x_{2}, x_{3}\right)=0$ for $\Sigma_{1}$ and $F_{2}\left(Q_{1}, Q_{2}, Q_{3}\right)=0$ for $\Sigma_{2}$. The touching manifold $M^{5} \subset\left(S E(3) \times \Sigma_{2}\right) \times \Sigma_{1}$ consists of solutions for the 7 equations in 12 variables

$$
F_{1}(X)=0, F_{2}(Q)=0, g \cdot Q=X, \text { and } \nabla F_{1}(X) /\left|F_{1}(X)\right|=\nabla F_{2}\left(g^{-1} Q\right) .
$$

We have 5 effective equations as the last three count as two. In the sequel we will identify $M^{5} \equiv \Sigma_{1} \times S O(3)$, where the first component is the contact point, and the second component the attitude matrix of the rolling body. Given $q_{1} \in \Sigma_{1}$ and $R \in S O(3)$ we can find the corresponding point $Q_{2} \in \Sigma_{2}$ and the position $x=x_{C M}$ of the center of mass. Indeed, since the normals are aligned, we have $N_{1}\left(q_{1}\right)=-R N_{2}\left(Q_{2}\right)$, where we orient $\Sigma_{2}$ with the exterior normal. For visualization convenience, we orient $\Sigma_{1}$ in such a way that at the contact points the normals point in opposite ways (for instance, in the sphere-sphere case, both are the exterior normals). Hence

$$
\begin{gathered}
Q_{2}=N_{2}^{-1}\left(-R^{-1} N_{1}\left(q_{1}\right)\right) \\
x=x_{C M}=q_{1}-R Q_{2}=q_{1}-R\left(N_{2}^{-1}\left(-R^{-1} N_{1}\left(q_{1}\right)\right)\right)
\end{gathered}
$$

(in the sequel we may drop the suffixes when no confusion may arise).

As found long ago by Euler, the kinetic energy of a rigid body $\mathcal{B}$ is given by

$$
2 T(x, R, \dot{x}, \dot{R})=\mu\|\dot{x}\|^{2}+I_{1} \Omega_{1}^{2}+I_{2} \Omega_{2}^{2}+I_{3} \Omega_{3}^{2},
$$

where $\mu$ is the total mass, $I_{j}$ the moments of inertia about the principal axis $e_{1}, e_{2}, e_{3}$ attached at the center of mass $x$, and $\Omega=\left(\Omega_{1}, \Omega_{2}, \Omega_{3}\right)^{t}$ the angular velocity vector written the body frame. More precisely, $R^{-1} \dot{R}=[\Omega]$ is the skew symmetric matrix

$$
[\Omega]=\left(\begin{array}{lll}
0 & -\Omega_{3} & \Omega_{2} \\
\Omega_{3} & 0 & -\Omega_{1} \\
-\Omega_{2} & \Omega_{1} & 0
\end{array}\right)
$$

See Arnold [1] for lower/upper case notations: The angular velocity in space is $\dot{R} R^{-1}=[\omega]$, with $\omega=R \Omega$.

There is no coupling between the translational and the rotational motions if the body is moving freely in space.

What is the dynamics when we impose the (holonomic) constraint of skiding? For simplicity, we ignore potential forces. In order to compute the Lagrangian

$$
T\left(q_{1}, R, \dot{q}_{1}, \dot{R}\right) \text { on } Q=\Sigma_{1} \times S O(3)
$$

governing the holonomical system defined by the touching conditions 2.12 .2 , we replace in 2.3 the velocity $\dot{x}$ of the center of mass by

$$
\dot{x}=\dot{q}_{1}-D_{\left(R, q_{1}\right)}\left[R\left(N_{2}^{-1}\left(-R^{-1} N_{1}\left(q_{1}\right)\right)\right)\right]\left(\dot{R}, \dot{q}_{1}\right) .
$$

This task is not so simple, because we need the derivative of the map

$$
F:\left(R, q_{1}\right) \in S O(3) \times \Sigma_{1} \mapsto R\left(N_{2}^{-1}\left(-R^{-1} N_{1}\left(q_{1}\right)\right)\right) \in \mathbf{R}^{3}
$$

appearing in 2.5. This notwithstanding, all the geometric information we need is contained in the Gauss maps:

Lemma 1

$$
d F_{\mid\left(R, q_{1}\right)} \cdot\left(\dot{R}, \dot{q}_{1}\right)=\omega \times\left(q_{1}-x\right)+\left(d N_{g \Sigma_{2}}\right)^{-1}\left(\omega \times N_{1}\left(q_{1}\right)-d N_{1}\left(q_{1}\right) \cdot \dot{q}_{1}\right)
$$

Proof. This is a simple exercise on Advanced Calculus. Here the rotational velocity $\dot{R}$ is written in the space frame, $\dot{R} R^{-1}=[\omega]$. Denoting by $g(t) \cdot \Sigma_{2}$ the current (located) position of the moving body, $g=(R, x) \in S E(3)$, we can reinterpret some objects that appear in the derivation, for instance $R^{-1} d N_{2}\left(Q_{2}\right) R=d N_{g \Sigma_{2}}\left(q_{1}\right)$. 
Example: sphere-sphere skiding. Let $\Sigma_{1}=S_{a}$ a sphere of radius $a$ centered at the origin, $\Sigma_{2}=S_{b}$ a sphere of radius $b$ rolling over $\Sigma_{1}$. Then (2.2) becomes as expected to $x=(1 \pm b / a) q_{1}$ The plus sign corresponds to the external case. The Lagrangian is

$$
2 T=\mu(1 \pm b / a)^{2}\left\|\dot{q}_{1}\right\|^{2}+I_{1} \Omega_{1}^{2}+I_{2} \Omega_{2}^{2}+I_{3} \Omega_{3}^{2}
$$

and the dynamics uncouple. Solutions are great circles in the sphere and Euler rigid body motion for the moving ball.

Proposition 3 For the problem of a convex body $\mathcal{B}$ with surface $\Sigma_{2}$ skiding over a sphere $\Sigma_{1}=S_{a}$ there is $S O(3)$ symmetry. We have a principal bundle structure with total space $Q=S_{a} \times S O(3)$,

$$
S O(3) \hookrightarrow Q \rightarrow Q / S O(3), R \cdot(q, S)=(R(q), R S)
$$

given by the diagonal action.

Along the fiber $\mathcal{B}$ is been moved rigidly rotations around the center of sphere $S_{a}$. In a sense motion along the fiber is "pure" skiding. The base of the bundle is the sphere $S_{a}$ and we can normalize to the unit sphere, parametrized by $\gamma$. There is a global section, namely $q_{1}=a \gamma \in S^{2} \mapsto(a \gamma, I) \in S_{a} \times S O(3)$. Notice that the induced global trivialization is not given just by the direct product structure. Fibers are diffeomorphic to $S O(3)$ via $R \mapsto(a R(\gamma), R)$. Along the global section, $q_{1}=a \gamma$ is the contact point in $\Sigma_{1}=S_{a}, Q_{2}=N_{2}^{-1}(-\gamma)$ is the corresponding contact point in $\Sigma_{2}$ (in its standard position). Given a curve $R(t) \in S O(3), R(0)=I, \dot{R}(0)=[\rho]$,

$$
V(\rho)=\frac{d}{d t}{ }_{t=0} R(t)(a \gamma, I)=(a \rho \times \gamma, \rho)
$$

gives a vertical vector.

Remark 2 Reduced equations for the skiding dynamics can be obtained by Marsden-Weinstein procedure [50]. For the symplectic reduction we need the momentum mapping $J: T^{*} Q \rightarrow \mathbf{R}^{3}$. An element of $T^{*} Q$ is of the form $\left(p_{R}, p_{q_{1}}\right)$, where $p_{R} \mapsto m \in s O(3)^{*} \equiv \mathbf{R}^{3}$ via right translation to the identity, and $p_{q_{1}} \in T^{*}\left(S_{a}\right)$ can be represented by a vector $r$ perpendicular to $q_{1}$. Using the abstract nonsense rule " $J$ of $p$ at $q$, on $X=p$ at $q$ on $V(X)$ " we get

$$
J: T^{*}\left(S_{a} \times S O(3)\right) \rightarrow s O(3)^{*}\left(\equiv \mathbf{R}^{3}\right), J\left(p_{R}, p_{q}\right)=m+q_{1} \times r=\ell .
$$

The six dimensional reduced symplectic manifolds are $M^{6}=J^{-1}(\ell) / S^{1}$, where $S^{1}$ denotes the isotropy group of $\ell$.

\subsection{No slip and no-twist constraints: kinematical relations for the Poisson vector}

The Poisson vector

$$
\gamma=R^{-1} N_{1}\left(q_{1}\right)=-N_{2}\left(Q_{2}\right)
$$

is the normal vector to $\Sigma_{1}$ seen in the body frame and is the basic object for rubber rolling. It is related to $Q_{2}$ by minus the Gauss map. We get immediatelly from $R \dot{Q}_{2}=\dot{q}_{1}$ :

Lemma 2 Kinematical relations for the Poisson vector, no slip:

$$
\dot{\gamma}+\Omega \times \gamma=R^{-1} d N_{1}\left(q_{1}\right) \cdot \dot{q}_{1}=\left[R^{-1} d N_{1}\left(q_{1}\right) R\right] \cdot \dot{Q}_{2} .
$$

In this formula, $q_{1}=\left(N_{1}\right)^{-1}(R \gamma)$ when the local inversion is possible 6 . In particular, if $\Sigma_{1}$ is a sphere of radius $a$ or a plane $(a=\infty)$, the term $R^{-1} d N_{1}\left(q_{1}\right) R$ is just $1 / a$ times the identity, the kinematical relation becomes

$$
\dot{\gamma}+\Omega \times \gamma=R^{-1}\left(\dot{q}_{1} / a\right)=\frac{1}{a} \dot{Q}_{2}=-\frac{1}{a} d\left(N_{2}\right)_{\gamma}^{-1} \cdot \dot{\gamma}
$$

Furthermore, if $\Sigma_{2}$ is also a sphere, of radius $b>0$,

$$
\dot{\gamma}+\kappa \Omega \times \gamma=0, \kappa=\frac{a}{a \pm b}
$$

where the plus sign corresponds to the external case, minus when one of the spheres contain the other. We can use only the plus sign and allow $b$ to be negative to represent the internal case (if $b<0$ and $|b|>a$ then the fixed sphere is inside the rolling sphere).

${ }^{6}$ Points on $\Sigma_{1}$ where one of the principal curvatures vanish may be specially relevant for the dynamics. 


\subsection{Rubber connection and curvature}

Imposing the no-slip constraints produces a map

$$
\omega \mapsto \dot{q}_{1}\left(\text { at } q_{1}\right)
$$

which gives a distribution of 3 -subspaces in $Q$. To rule out twisting, one adds

$$
\omega \cdot N_{1}\left(q_{1}\right)=0 \text { or equivalently } \Omega \cdot \gamma=0 \text {. }
$$

In more detail, the no-slip constraint in $Q=\Sigma_{1} \times S O(3)$ follows by replacing the left-hand side $\dot{x}$ in 2.5 by

$$
\dot{x}=\omega \times\left(x-q_{1}\right) .
$$

In view of lemma 1 the result is

$$
\dot{q}_{1}=d N_{g \Sigma_{2}}^{-1}\left(\omega \times N_{1}\left(q_{1}\right)-d N_{1}\left(q_{1}\right) \cdot \dot{q}_{1}\right)
$$

or, in a more symmetric form,

$$
\left[d N_{g \Sigma_{2}}+d N_{1}\right]\left(q_{1}\right) \cdot \dot{q}_{1}=\omega \times N_{1}\left(q_{1}\right)=\omega \times\left(-R N_{2}\left(Q_{2}\right)\right) .
$$

Since for no-slip the tangent vectors of curves $q_{1}(t)$ and $Q_{2}(t)$ correspond, $R \dot{Q}_{2}=\dot{q}_{1}$, equation 2.19 gives

$$
\Omega \times \gamma=R^{-1}\left[d N_{g \Sigma_{2}}+d N_{1}\right]\left(q_{1}\right) \cdot \dot{q}_{1}=d N_{2}\left(Q_{2}\right) \cdot \dot{Q}_{2}+d N_{1}\left(q_{1}\right) \cdot \dot{q}_{1} .
$$

Imposing in addition to 2.19 the no-twist constraint 2.16) allows us to solve for $\omega$ in terms of $\dot{q}_{1}$.

Lemma 3 The "rubber connection":

$$
\omega=N_{1}\left(q_{1}\right) \times\left(\left[d N_{g \Sigma_{2}}+d N_{1}\right]\left(q_{1}\right) \cdot \dot{q}_{1}\right.
$$

This formula defines an Ehresmann connection on the bundle $S O(3) \times \Sigma_{1} \rightarrow \Sigma_{1}$. Equation (2.20) can be rewritten in the body frame as

$$
\Omega=\left[\left(I d+\left(R^{-1} d N_{1} R\right)\left(d N_{2}\right)^{-1}\right)\right](\dot{\gamma}) \times \gamma
$$

Note that there is no $S O(3)$ equivariance unless $\Sigma_{1}$ is a sphere.

Consider a small curve $C_{1}$ around a point $q_{1} \in \Sigma_{1}$. The curvature of the Ehresmann connection is the limit

$\lim$ ( how much a frame attached to $\Sigma_{2}$ rotates ) / ( area inside the curve in $\left.\Sigma_{1}\right) \quad$, as $C_{1}$ shrinks .

We have computed the curvature for the case of a sphere rolling over another sphere, see section 3.3 below. In order to appreciate the difficulties of the calculation, we challenge the reader to attempt computing by brute force the curvature in the case of a surface of revolution rolling over a sphere. We claim, however, that it is possible to give a formula for the Ehresmann curvature of the rubber connection in the case of general surfaces $\Sigma_{1}, \Sigma_{2}$ in terms of natural geometric objects. Accepting at face value the information that rubber rolling belongs to the intrinsic geometry [14], the idea is to use proposition 2 together with the Gauss-Bonnet theorem. The result (see details in the companion paper [29]) is a kind of dynamical "Egregium theorem":

Theorem 2 (Bryant and Hsu [14]). Curvature of Ehresmann connection, general case: Let $k_{i}\left(q_{i}\right)$ the Gauss curvature of $\Sigma_{i}$ at the corresponding points $q_{i}$, such that $q_{1}=g q_{2}, g=(R, a) \in S E(3)$. Then

$$
K=\left(1-\frac{k_{1}\left(q_{1}\right)}{k_{2}\left(q_{2}\right)}\right) k_{1}\left(q_{1}\right) d \Sigma_{1} .
$$

where $d \Sigma_{1}$ is the area form of $\Sigma_{1}$.

This result is remarkable because the connection 1-form already involves the derivative of the Gauss maps, so at first sight the Ehresmann curvature should involve second derivatives.

Remark 3 Koon and Marsden have shown that a key ingredient for the equations of motion for nonholonomic systems is the curvature of the local Ehresmann connection associated to a splitting of a coordinate system (see [6] 43]). Both for marble or rubber rolling this splitting is global. See Theorem 3 below for the case when $\Sigma_{1}$ is a sphere. 


\subsection{Equations of motion: general physics approach}

In this section we follow Borisov and Mamaev [10] closely?. In addition to the kinematical relation (2.12), three differential equations for the rolling body can be derived from

$$
\dot{\ell}_{C M}=\tau_{C M},
$$

where $\ell_{C M}, \tau_{C M}$ are respectively the angular momentum and the torque of the external forces with respect to the center of mass.

In basic mechanics textbooks it is sometimes overlooked that the above formula does not hold in general if one takes $q_{1}$ as the base point instead of the center of mass $C M$. Recall that the angular momentum with respect to the contact point $q_{1}$ and with respect to the center of mass $x$ are related by

$$
\ell_{q_{1}}=\left(x-q_{1}\right) \times \mu \dot{x}+\ell_{C M} .
$$

Differentiating, we get

$$
\dot{\ell_{q_{1}}}=\left[\left(x-q_{1}\right) \times f_{e x t}+\tau_{C M}\right]+\frac{d}{d t}\left[\left(x-q_{1}\right)\right] \times \mu \dot{x},
$$

and we recognize the term $\left(x-q_{1}\right) \times f_{e x t}+\tau_{C M}$ as the torque about the contact point. The correct form of the torque equation is

$$
\dot{\ell_{q_{1}}}=\tau_{q_{1}}+\frac{d}{d t}\left[\left(x-q_{1}\right)\right] \times \mu \dot{x} .
$$

containing an ammended term, which can be called the dynamical torque,

$$
\tau_{d y n}=\frac{d}{d t}\left[\left(x-q_{1}\right)\right] \times \mu \dot{x} .
$$

The non-sliping condition implies $\dot{x}=\omega \times\left(x-q_{1}\right)$, so in the space frame

$$
\dot{\ell_{q_{1}}}=\tau_{q_{1}}+\frac{d}{d t}\left[\left(x-q_{1}\right)\right] \times \mu \omega \times\left(x-q_{1}\right) .
$$

If we go to the body frame, we get 8

$$
\dot{L}+\Omega \times L+\left(\Omega \times Q_{2}\right) \times\left(\mu \dot{Q}_{2}\right)=T
$$

where we recall for clarity, that

$$
\ell_{q_{1}}=R L, x-q_{1}=R Q_{2}, \omega=R \Omega, \tau=R T .
$$

In order to obtain a linear relation between $L$ and $\Omega$ (depending on the Poisson vector $\gamma$ ), we rewrite the total energy of the system as

$$
2 H=2 T=\left(\ell_{q_{1}}, \omega\right)=(L, \Omega), \text { whith } R L=\ell_{q_{1}},
$$

where $\ell_{q_{1}}$ is the angular momentum with respect to the contact point viewed in space, $L$ its coordinates in the body frame. Substituting the no-slipping constraint in 2.3, , one gets

$$
L=A \Omega+\mu Q \times(\Omega \times Q), A=\operatorname{diag}\left(I_{1}, I_{2}, I_{3}\right) .
$$

$L=\frac{\partial T}{\partial \Omega}$, is the angular momentum of the rolling body with respect to the contact point, seen in the body frame. Relation (2.28) can be inverted,

$$
\Omega=\left(A+\mu\|Q\|^{2} i d\right)^{-1} L+\alpha(L, Q)\left(A+\mu\|Q\|^{2} i d\right)^{-1}(Q)
$$

where

$$
\alpha(L, Q)=\mu \frac{\left(Q,\left(A+\mu\|Q\|^{2} i d\right)^{-1} L\right)}{1-\mu\left(Q,\left(A+\mu\|Q\|^{2} i d\right)^{-1} Q\right)} .
$$

It will be convenient to use the shorthand $\tilde{A}=A+\mu\|Q\|^{2} i d$.

Proposition 4 (Counting dimensions and equations) In the no-slip case we have an eight dimensional phase-space. Using $(R, \gamma, L) \in S O(3) \times S^{2} \times \mathbf{R}^{3}$ as coordinates (we are assuming that $q_{1}=\left(N_{1}\right)^{-1}(R \gamma)$ can be locally inverted), the dynamics is given by the momentum equation (2.27), the kinematic relations (2.12) and the attitude equation $\dot{R}=R[\Omega]$. We will have a closed system of equations since $\Omega$ is a function of $(L, \gamma)$ via formula (2.29). If we add the no-twist constraint, the phase space is seven dimensional: one adds the constraint $(\Omega, \gamma)=0$, and uses it to eliminate the unknown torque around the normal.

Reconstruction of $q_{1}$ motion in $\Sigma_{1}$ can be done either via $\dot{q}_{1}=R \dot{Q}_{2}$, (where as we recall, $Q$ is related to $\gamma$ via the Gauss map) or simply inverting $N_{1}\left(q_{1}\right)=R \gamma$. In general, the kinematical relation couples $R$ with the other variables, but when $\Sigma_{1}$ is a plane or a sphere, the attitude equation decouples from the equation for $\gamma$ and $L$, a consequence of $S O(3)$ symmetry.

\footnotetext{
${ }^{7}$ For more abstract approaches, see eg., [42], [46],[47].

${ }^{8}$ This is precisely eqs. (1.1) of [10] in our notation.
} 
Remark 4 If $x$ and $q_{1}$ move in such a way that a combination of them with fixed coefficients remains constant, then $\dot{x}$ and $\dot{q}_{1}$ are proportional. Consequently, the ammended term in 2.25 vanishes identically. For instance, this happens in the case of spheres rolling over spheres (or a plane 9 .

Sphere-sphere rubber rolling. As the dynamical torque is identically zerd 10 , we are left with

$$
\dot{L}+\Omega \times L=\tau \gamma, \dot{\gamma}=\kappa \gamma \times \Omega, L=\tilde{A} \Omega+\mu b^{2}(\Omega, \gamma) \gamma=\tilde{A} \Omega \text {, with }(\Omega, \gamma)=0
$$

with $\kappa=a /(a+b)$. The right hand side $T$ is the external torque about the contact point in the body frame (zero for the no-slip case, but twisting allowed.) In view of the no-twist constraint $(\Omega, \gamma)=0$ it is immediate that $\Omega=\tilde{A}^{-1} L$, and we get the following expression for the multiplier:

$$
\tau\left(\gamma, \tilde{A}^{-1} \gamma\right)=\left(\tilde{A}^{-1} L \times L, \tilde{A}^{-1} \gamma\right)+\kappa\left(\tilde{A}^{-1} L, \tilde{A}^{-1} L \times \gamma\right)
$$

The problem of a rubber ball rolling on a plane corresponds to $\kappa=1$ and was discussed briefly in [27]. We showed the existence of an invariant measure

$$
\mathrm{v}=F(\gamma)^{-1 / 2} d L_{1} d L_{2} d L_{3} d \gamma_{1} d \gamma_{2} d \gamma_{3}
$$

with

$$
F=I_{1} I_{2} I_{3}\left(\left(A^{-1} \gamma, \gamma\right)+\mu b^{2}\left[\frac{\gamma_{2}^{2}+\gamma_{3}^{2}}{I_{2} I_{3}}+\frac{\gamma_{1}^{2}+\gamma_{3}^{2}}{I_{1} I_{3}}+\frac{\gamma_{1}^{2}+\gamma_{2}^{2}}{I_{1} I_{2}}\right]+\frac{\mu^{2} b^{4}}{I_{1} I_{2} I_{3}}\right) .
$$

Borisov and Mamaev [12] called the attention that the solutions of the rubber ball on the plane can be set into correspondence with solutions of Veselova's system [61,60] namely, a rigid body with one right invariant constraint (formally, $b=0$ ). They used a trick that seems to go back to Chaplygin, which is really a "Columbus egg": one takes suitable linear combinations of $\gamma$ and $L$ and show that the equations of motion correspond. Unfortunately, this clever maneuver only seems to work when $\kappa=1$.

\subsection{Marble Chaplygin sphere over a sphere or a plane}

This section is intended as background material, just for comparison with the rubber rolling problem discussed next. For more details on what is known about marble rolling of convex bodies over a plane or a sphere, see [10].

In 1903 Chaplygin [22] integrated using hyperelliptic functions the following system for $(L, \gamma) \in \mathfrak{R}^{3} \times \mathfrak{R}^{3}$ :

$$
\begin{aligned}
& \dot{L}+\Omega \times L=0, \\
& \dot{\gamma}+\Omega \times \gamma=0,
\end{aligned}
$$

where $\Omega$ is defined by 2.29 with $Q=r \gamma$, namely,

$$
\Omega(L, \gamma)=\left(A+\mu r^{2} i d\right)^{-1} L+\alpha(L, \gamma)\left(A+\mu r^{2} i d\right)^{-1}(\gamma)
$$

with

$$
\alpha(L, \gamma)=\mu r^{2} \frac{\left(\gamma,\left(A+\mu r^{2} i d\right)^{-1} L\right)}{1-\mu r^{2}\left(\gamma,\left(A+\mu r^{2} i d\right)^{-1} \gamma\right)} .
$$

They describe the motion of a marble ball of radius $r$ and mass $\mu$, moments of inertia $I_{j}$ about the geometric center (which is also the center of mass), rolling without sliping over a plane. Twisting motions (rotations about the vertical) are allowed. $L$ is the angular momentum of the ball with respect to the contact point, $\Omega$ the angular velocity and $\gamma$ the vertical vector, all viewed on a reference frame attached to the ball.

This system has four independent integrals

$$
f_{1}=(L, \Omega), f_{2}=(L, L), f_{3}=(\gamma, \gamma), f_{4}=(L, \gamma)
$$

where $2 f_{1}$ is the energy and $f_{4}$, called the area integral, is the third spacial component of the angular momentum. Topologically, the common level sets of these integrals are tori; Chaplygin showed that there is a smooth invariant measure $[F(\gamma)]^{-1 / 2} d \gamma d L$, with

$$
F(\gamma)=\frac{1}{\mu r^{2}}-\left(\gamma, \tilde{A}^{-1} \gamma\right), \tilde{A}=A+\mu r^{2} i d
$$

Integrability follows from the celebrated Jacobi's last multiplier method, which says that an ODE on a two dimensional manifold having a smooth invariant measure can be solved by quadratures. Detailed remakes of Chaplygin's paper appeared recently, see Kilin [37] and Duistermaat [26]. The planar motion can be reconstructed by integrating $\dot{x}=r \omega(t) \times k$.

\footnotetext{
${ }^{9}$ Historical note: Chaplygin seems to have been the first one to discuss geometrical conditions under which the second term vanishes. See [53 2] 41] for discussions on this often neglected issue.

${ }^{10}$ For abstract explanations, see discussions on nonholonomic Noether theorem in [2 56].
} 
Remark 5 The issue whether Chaplygin marble system is hamiltonizable or not has been discussed in [8. 9. 26]. Perhaps due to some misprints we could not verify the Borisov-Mamaev bracket, but we are glad to know that it has been verified and explained independently by Naranjo[52]. Theoretical issues about almost Poisson brackets are outside the scope of our paper, but we believe will be much in evidence in the next years, see [15. 16. 47. 48] for background.

To extend Chaplygin's sphere-plane equations to the sphere-sphere case, it suficies to replace equation (2.36) by the kinematical relation

$$
\dot{\gamma}+\kappa \Omega \times \gamma=0
$$

with $\kappa=a /(a+b)$ (Recall that $b>0$ in the external case, so $0<\kappa<1$; in the internal case where $b<0$ and $|b|<a$, $\kappa>1$; and $\kappa=1$ for rolling over a plane $(a \rightarrow \infty)$. One can also consider the case $-\infty<\kappa<0$, when the fixed ball $S_{a}$ lives inside the rolling ball $S_{|b|}, b<0,|b|>a$.)

The formula for the energy is the same as in the sphere-plane case,

$$
2 H=2 T=\mu b^{2}\|\Omega \times \gamma\|^{2}+I_{1} \Omega_{1}^{2}+I_{2} \Omega_{2}^{2}+I_{3} \Omega_{3}^{2} .
$$

We now give an argument to explain why the invariant measure stays the same, independently of the value of $\kappa$.

Proposition 5 There is a smooth invariant measure for the marble rolling of a sphere over a sphere. The density function is the same as in the case of Chaplygin's sphere rolling over a plane, $F(\gamma)^{-1 / 2}$, with $F$ given by (2.40).

Proof. As a warm up, let us verify first that the total energy function 2.42) of the sphere rolling over a sphere (a formula in which the radius of the base sphere does not appear) is conserved along the trajectories of 2.35, 2.41). This direct check will be instructive, since all sphere-sphere systems have the same energy function and the same $\dot{L}$ equation, but a family of $\dot{\gamma}$ equations, parametrized by $\kappa$. We still get $\dot{H}=\operatorname{grad}_{(L, \gamma)} H \cdot(\dot{L}, \dot{\gamma}) \equiv 0$ because both

$$
\begin{aligned}
& \nabla_{L} H \cdot \dot{L} \equiv 0, \\
& \nabla_{\gamma} H \cdot \dot{\gamma} \equiv 0 .
\end{aligned}
$$

Equation 2.43) is immediate, since $\nabla_{L} H=\Omega$ and $\dot{L}=\Omega \times L$ is perpendicular to $\Omega$. Now, we know that the proposition holds for the case $\kappa=1$. Equation (2.44) must be therefore true for $\kappa=1$. Now, for an arbitrary $\kappa$, the left hand side of (2.44) only picks this multiplicative factor so it must also be zero and there is no need to do the computation).

In order to verify that the measure is invariant, we follow a similar procedure. Let us scrutinize the derivation presented Duistermaat, [26] (lemma 7.1) for the case $\kappa=1$. His approach was to split

$$
\operatorname{div}_{(\gamma, L)}(\gamma \times \Omega(\gamma, L), L \times \Omega(\gamma, L))
$$

as the trace of the derivative with respect to $\gamma$ of the first component plus the trace of the derivative with respect to $L$ of the second component. The latter trace is obviously identically zero, whereas the first one is found to be (see (7.2) in that paper)

$$
\left.\operatorname{div}_{\gamma}(\gamma \times \Omega(\gamma, L))\right)=\frac{\left(\gamma \times \tilde{A}^{-1} \gamma, \tilde{A}^{-1} L\right)}{F(\gamma)}
$$

On the other hand, the directional derivative of $F(\gamma)$ is found to be

$$
(\operatorname{grad} F, \gamma \times \Omega(\gamma, L))=2\left(\gamma \times \tilde{A}^{-1} \gamma, \tilde{A}^{-1} L\right)
$$

and hence

$$
\operatorname{div}_{(\gamma, L)}\left[F^{-1 / 2}(\gamma \times \Omega(\gamma, L), L \times \Omega(\gamma, L))\right]=\operatorname{div}_{\gamma}\left[F^{-1 / 2} \gamma \times \Omega(\gamma, L)=0\right.
$$

Now, comes the argument: the only change caused by the presence of $\kappa$ is to multiply both (2.45) and 2.46) by this same fator, so the net result stays zero.

Functions $f_{1}, f_{2}, f_{3}$ are still integrals of motion for any $\kappa$, but the "area integral" $f_{4}$ is lost! It is easy to see that it holds only for $\kappa=1$. In the three dimensional manifolds $M^{3}$ defined by level sets of the three (surviving) integrals we have an invariant measure. Flows on three dimensional compact manifolds with an invariant measure are of interest for dynamicists and one expect that the motion will be ergodic in $M^{3}$ except perhaps for some special values of $k$, where some new integral of motion may appear.

Borisov found the only known (perhaps unique) new integrable case, $\kappa=-1$, corresponding to an internal fixed ball of half the radius of the rolling ball, see [10], pag. 194.

Remark 6 The external case with equal radii (corresponding to $\kappa=1 / 2$ ) could be (erroneously) thought to be also integrable, for a simple reason. As we will see below, the two dimensional rubber distribution is holonomic for two spheres of equal radius. One could hope that relaxing the no-twist constraint would keep the distribution holonomic, as it amounts to allow any finite rotation of the moving sphere about the normal at any given (but arbitrary) point on the base sphere. Each leaf $\mathcal{F}$ would then be three dimensional, forming a a $S^{1}$ bundle over $S^{2}$, and the topology would be (probably) that of $S U(2)$. Unfortunately there is a serious blunder is this reasoning. Although $\operatorname{span}\left(X_{1}, X_{2}\right)$ is integrable, adding the infinitesimal rotations about the contact points produces a 3 dimensional distribution $\operatorname{span}\left(X_{1}, X_{2}, X_{3}\right)$ with growth 3-5. 


\section{Rubber rolling over a sphere as a $S O(3)$ Chaplygin system.}

\subsection{Reduction to $T^{*} S^{2}$.}

Consider the principal bundle with total space $Q=S_{a} \times S O(3)$,

$$
S O(3) \hookrightarrow Q \rightarrow Q / S O(3), R \cdot(q, S)=(R(q), R S)
$$

given by the diagonal action. It is not hard to see that the no-slip and no-twist constraints define a principal bundle connection for any rolling surface $\Sigma_{2}$. We call it the rubber connection.

Recall (2.20), the Ehresmann connection for the rubber rolling of a surface $\Sigma_{2}$ on an arbitrary base $\Sigma_{1}$ :

$$
\omega=N_{1}\left(q_{1}\right) \times\left(\left[d N_{g \Sigma_{2}}+d N_{1}\right]\left(q_{1}\right) \cdot \dot{q}_{1} .\right.
$$

When $\Sigma_{1}$ is a sphere there is an extra feature, namely, equivariance, so we have a principal bundle connection. We need to show that if $\omega$ solves both equations 2.16, 2.19, which in this case become

$$
\omega \times q_{1}=\left[I+a d N_{g \Sigma_{2}}\left(q_{1}\right)\right] \dot{q}_{1}, \omega \cdot q_{1}=0,
$$

then $R \omega$ solves

The second fact is obvious, and the first follows from 11

$$
R \omega \times R q_{1}=\left[I+a d N_{R g \Sigma_{2}}\left(R q_{1}\right)\right]\left(R \dot{q}_{1}\right), \quad \mathbf{R} \omega \cdot R q_{1}=0 .
$$

$$
d N_{R g \Sigma_{2}}\left(R q_{1}\right)=R d N_{g \Sigma_{2}}\left(q_{1}\right) R^{-1} .
$$

See [39] for reduction written in Lagrangian form. The reduction procedure in Hamiltonian form, following the recipe given in [27] is as follows:

Theorem 3 Let $\Sigma_{2}=\Sigma$ be arbitrary but $\Sigma_{1}$ a sphere of radius a. Then rubber rolling is described by a non-abelian Chaplygin system on the bundle $Q=S O(3) \times S^{2}(a)$, with symmetry group $S O(3)$ acting diagonally. The constraints are given by the rubber connection

$$
\omega=\frac{q_{1}}{a} \times\left[d N_{g \Sigma_{2}} \cdot \dot{q}_{1}+\frac{\dot{q}_{1}}{a}\right] .
$$

Define the projection is by $\pi(R, q)=R^{-1} q / a \in S^{2}, R \in S O(3), q \in S^{2}(a)$. Then the dynamics reduces to an almost-symplectic system $\left(H, \omega_{N H}\right)$ in $T^{*} S^{2}$. The Hamiltonian $H$ in $T^{*} S^{2}$ is the Legendre transform of the compressed Lagrangian $T(\gamma, \dot{\gamma})$ given by

$$
2 T=(\tilde{A} \Omega, \Omega) \text { with } \Omega=\left[\left(I d+\frac{1}{a}\left(d N_{2}\right)^{-1}(\gamma)\right)\right](\dot{\gamma}) \times \gamma, \tilde{A}=A+\mu\|Q\|^{2} i d, Q=-N_{\Sigma}^{-1}(\gamma)
$$

obtained from the original one by horizontal lifting via the connection. The 2-form is given by $\omega_{n h}=\omega_{\text {can }}^{T^{*} S^{2}}+(J, K)$ and in general is non closed. The ammended term $(J, K)$ is semibasic; $J$ is the momentum (2.10) of the SO(3) action and $K$ is the curvature (2.22) of the rubber connection.

The domains of $J$ and $K$ are matched by the Legendre transformation from $T Q$ to $T^{*} Q$. Their Ad and Ad*ambiguities cancel out automatically. In some more detail,

$$
(J, K)=\left(1-\frac{1 / a^{2}}{k_{\Sigma}(\gamma)}\right)(\gamma, M) d \operatorname{area}_{S^{2}}
$$

where $\gamma=q /$ a is the contact point in the base sphere, $k_{\Sigma}(\gamma)$ is the Gauss curvature of the rolling surface at the current contact point $Q=-N_{\Sigma}^{-1}(\gamma)$, and $M=A \Omega$ is the angular momentum at the center of mass of the rolling body.

We plan to use this result as a basis for future work. The first in line is the rubber rolling over a sphere of surfaces of revolution with two equal inertias. For the rest of this paper we restrict ourselves to the sphere-sphere example.

\subsection{The rubber sphere-sphere connection 1-form.}

We show that for the sphere-sphere problem there is an invariant measure, and even better, the reduced system is conformally symplectic12. The expert can skip many of the redundant calculations below, intended as checks of the general results presented earlier. When $\Sigma_{2}$ is a sphere of radius $b, 3.1$ becomes

$$
\omega=\frac{1}{a^{2}}\left(1 \pm \frac{a}{b}\right) q_{1} \times \dot{q}_{1}
$$

Recall that for simplicity we drop the \pm sign, using the convention that $b$ is negative one of the spheres contains the other.

\footnotetext{
${ }^{11}$ Latexing is more difficult than the result. A drawing should make it obvious.

${ }^{12} \mathrm{We}$ conjecture that this is also true for bodies of revolution.
} 
Lemma 4 The horizontal lift of $\dot{\gamma} \in T_{\gamma} S^{2}$ at $(a R(\gamma), R) \in Q$ is given by

$$
\dot{\gamma} \mapsto \operatorname{Hor}(\dot{\gamma})=\left(-b R(\dot{\gamma}),\left(1+\frac{b}{a}\right)\left(L_{R}\right)_{*}[\dot{\gamma} \times \gamma]\right) .
$$

where $\left(L_{R}\right)_{*}$ indicates the left translation and [,] the standard isomorphism from $\mathbf{R}^{3}$ to $s O(3)$.

Proof. The formula looks odd due to the fact that the $S O(3)$ action is diagonal. Note first that

$$
\left(\pi_{*}\right)_{q, R)}(\dot{q}, \dot{R})=\frac{1}{a}\left(R^{-1} \dot{q}-R^{-1} \dot{R} R^{-1} q\right) .
$$

Take $a R(\gamma)=q, \dot{q}=-b R(\dot{\gamma})$ and $\dot{R}=\left(L_{R}\right) *[\dot{\gamma} \times \gamma]$. Then

$$
\left.\left(\pi_{*}\right)_{(q, R)}(\dot{q}, \dot{R})=-\frac{b}{a} \dot{\gamma}-\left(1+\frac{b}{a}\right)(\dot{\gamma} \times \gamma) \times \gamma\right)=\dot{\gamma}
$$

and a short calculation shows that (3.4) holds:

$$
\frac{1}{a^{2}}\left(1+\frac{a}{b}\right) R^{-1}\left(q_{1} \times \dot{q}_{1}\right)=\frac{1}{a}\left(1+\frac{a}{b}\right) \gamma \times(-b \dot{\gamma})=\left(1+\frac{b}{a}\right) \dot{\gamma} \times \gamma
$$

In order to find the connection form, it sufficies to compute along the global section $\gamma \mapsto(a \gamma, I)$.

Proposition 6 The connection 1-form $\phi$ interpreted as a 1-form in $S^{2} \times S O(3)$ with values in $s 0(3) \equiv \mathbf{R}^{3}$, is given at the point $(\gamma, I)$ of the global section by

$$
\phi(\dot{\gamma}, \sigma)=(1+b / a) \gamma \times \dot{\gamma}-(b / a)[\sigma-(1+a / b)(\sigma \cdot \gamma) \gamma]
$$

Proof. By a direct check one verifies, as desired, that

$$
\left.\phi(\rho \times \gamma, \rho)=\rho, \phi\left(-\frac{b}{a}\right) \dot{\gamma},\left(1+\frac{b}{a}\right) \dot{\gamma} \times \gamma\right)=0 .
$$

While the metric is left invariant under the diagonal action of $G=S O(3)$ on $S_{a} \times S O(3)$, looking at (3.4) we observe that in the sphere-sphere problem the constraints are invariant under another action of $S O(3)$ on $S^{2} \times S O(3)$, namely the right action on the group component: $(\gamma, R) S=(\gamma, R S)$. This makes the problem reminiscent to a LR nonholonomic Chaplygin system $H \hookrightarrow G \rightarrow H / G$, see [31], except that here the bundle structure is of the form

$$
G \hookrightarrow G \times H / G \rightarrow H / G
$$

with $H \equiv S^{1}$ the rotations about the vertical axis, $H / G \equiv S^{2}$ the homogeneous coset space.

Assuming that results similar to those for LR systems would still hold, since the reduced dynamics has two degrees of freedom, we predict the existence of a function $f: S^{2} \rightarrow \mathbf{R}$ such that $d\left(f \omega_{n h}\right)=0$. In other words, the reduced system in $T^{*} S^{2}$ would be Hamiltonizable, since the nonholonomic 2-form is conformally symplectid 13 and in particular, there will be a smooth invariant measure. Actually, we guess $f$ from the density function of the invariant measure, relative to the Liouville volume of $T^{*} S^{2}$. The good news is that it is possible to verify directly if a nonholonomic system has a smooth invariant measure, see [4, $17,38,44,64$.

In future work we plan to pursue a LR theory for principal bundles of the type 3.7). Here, we use an alternative, albeit tentative approach: in order to make the LR analogy more plausible, we consider the whole "onion" of base spheres $S_{a}, a>0$ (the singular limit $a=0$ represents Veselova system). Then our mechanical system lives on an extended configuration space, $Q=E(3)^{\bullet}=S O(3) \times\left(\mathbf{R}^{3}-0\right)$ with a metric which is manifestly invariant under the left action of $S O(3)$ :

$$
2 T=\mu(1 \pm b /|q|)^{2}\|\dot{q}\|^{2}+I_{1} \Omega_{1}^{2}+I_{2} \Omega_{2}^{2}+I_{3} \Omega_{3}^{2}
$$

Note that the presence of $|q|$ in the denominator forces us to remove the origin of $\mathbf{R}^{3}$.

The pseudo LR system has bundle structure ${ }^{14}$

$$
S O(3)) \hookrightarrow E(3)) \rightarrow \mathbf{R}^{3}, R \cdot(S, q)=(R S, R q)
$$

We now rewrite the no-slip, no-twist conditions in terms of differential forms. The right and left invariant forms in $S O(3)$ will be denoted here

$$
d R R^{-1}=\left(\begin{array}{lll}
0 & -\rho_{3} & \rho_{2} \\
& 0 & -\rho_{1} \\
* & 0
\end{array}\right), R^{-1} d R=\left(\begin{array}{lll}
0 & -\lambda_{3} & \lambda_{2} \\
0 & -\lambda_{1} \\
* & 0
\end{array}\right) .
$$

\footnotetext{
${ }^{13}$ See $[32,33,59,63]$ to appreciate the consequences of being conformally symplectic.

${ }^{14}$ Using a more abstract language helps to generalize to configuration spaces that are semi-direct products of Lie groups.
} 
The distribution $\mathcal{D}$ of 2-dimensional subspaces in $E(3)^{\bullet}$ with $|q| \neq 0$, is the joint kernel of the four differential forms $\eta_{i}, 1 \leq i \leq 4$ in $E(3)$ defined by

$$
\left(\eta_{1}, \eta_{2}, \eta_{3}\right)^{t}=\vec{\rho}-\frac{1}{|q|^{2}}\left(1+\frac{|q|}{b}\right) q \times d q, \eta_{4}=q_{1} d q_{1}+q_{2} d q_{2}+q_{3} d q_{3} .
$$

where $\vec{\rho}=\left(\rho_{1}, \rho_{2}, \rho_{3}\right)^{t}$. Notice that the Pffafian equation $(q, \rho)=0$ defining the no-twist condition is satisfied automatically when $\eta_{i}=0,1 \leq i \leq 4$. Notice also that $S \in S O(3)$ acting on the right on an element $(R, q) \in S E(3)$ gives $(R S, q)$. This is best seen using the costumary representation for $E(3)$ :

$$
(R, q) \leftrightarrow\left(\begin{array}{cc}
R & q \\
0 & 1
\end{array}\right)
$$

This implies

Proposition 7 Consider the distribution $\mathcal{D}$ in $E(3)^{\bullet}$ defined by the constraints $\eta_{i}=0,1 \leq i \leq 4$. These forms are invariant under the right action of $S O(3)$ in $S E(3)^{\bullet}$, that is $\mathcal{D}_{(R, q)}=\mathcal{D}_{(I, q)} \cdot R$.

\subsection{Curvature of the sphere-sphere connection}

This section could be avoided in view of 2.22 but we decided to include it for completeness. We already know the connection 1 -form $\phi$ at the global section. In order to compute the curvature 2 -form we need an expression everywhere. This can be done by equivariance, but this is a somewhat unpleasant task $\left[\phi_{(q, R)}(\dot{q}, \dot{R})\right.$ is obtained from 3.6 by computing $R$ of the result of $\phi(\dot{\gamma}, \sigma)$ with the inputs $\gamma \leftarrow R^{-1} q / a, \dot{\gamma} \leftarrow R^{-1} \dot{q} / a-\omega \times \gamma,[\sigma] \leftarrow R^{-1} \dot{R}, \omega=\dot{R} R^{-1}$. Also, we must extend it to a connection in $\mathbf{R}^{\bullet} \times S O(3) \hookrightarrow S E(3)^{\bullet} \rightarrow S^{2}$, by adding a component $(\dot{q}, q /|q|)$ to $\phi$, and replacing $a$ by $|q|$ and $\dot{q}$ by $\dot{q}-\left(1 /|q|^{2}\right)(q, \dot{q})$. ]

After putting everything inside $\phi$, we would get an unpleasant formula. Computing the curvature directly would be a "tour de force". We prefer to guess the curvature by a wrong but algebraically elenant calculation. We then reinterpret and confirm the guess using elementary geometry.

We can use cartesian coordinates in $\mathbf{R}^{3}$, since we extended the base space to an "onion" of spheres. Consider the vectorfields in $S E(3)^{\bullet}$ given by

$$
Y_{1}=\partial / \partial x+\left(\frac{1}{a}+\frac{1}{b}\right)\left(\frac{z}{a} X_{2}-\frac{y}{a} X_{3}\right), Y_{2}=\partial / \partial y+\left(\frac{1}{a}+\frac{1}{b}\right)\left(-\frac{z}{a} X_{1}+\frac{x}{a} X_{3}\right)
$$

where $X_{1}, X_{2}, X_{3}$ are the right invariant vectorfields of $S O(3)\left(\left[X_{1}, X_{2}\right]=-X_{3}\right.$, etc). These vectors are horizontal at the point $q_{a}=(0,0, a), R=I$. A simple mental calculation gives us

$$
\begin{gathered}
{\left[Y_{1}, Y_{2}\right]_{\mid\left(q_{a}, I\right)}=\left[2 \frac{1}{a}\left(\frac{1}{a}+\frac{1}{b}\right)-\left(\frac{1}{a}+\frac{1}{b}\right)^{2}\right] X_{3},} \\
{\left[2 \frac{1}{a}\left(\frac{1}{a}+\frac{1}{b}\right)-\left(\frac{1}{a}+\frac{1}{b}\right)^{2}\right]=\frac{1}{a^{2}}-\frac{1}{b^{2}} .}
\end{gathered}
$$

Now, both $Y_{1}$ and $Y_{2}$ must be multiplied by $-b$ in order to be the horizontal lifts at $q_{a}$ of the unit vectors $(1,0,0)$ and $(0,0,1)$ at the north pole of $S^{2}=S^{2}(1)$, see equation 3.5 in lemma4 As a result, we have to multiply that result by $b^{2}$. From H.Cartan's magic formula, we get (note the extra - sign):

Proposition 8 The curvature of the connection, interpreted as a 2 form in the unit sphere $S^{2}$ with values in $\mathbf{R}^{3} \equiv s O(3)$, is

$$
K(\gamma)=\left(1-\frac{b^{2}}{a^{2}}\right) \gamma d \operatorname{area}_{S^{2}}
$$

This calculation can be made more rigorous. We forgot to compute $Y_{1} \phi\left(Y_{2}\right)-Y_{2} \phi\left(Y_{1}\right)$ in H. Cartan's "magic formula", at $(0,0, a)$. By a symmetry argument, one should be able to show that it must vanish.

We now present a geometric way to compute the curvature. Consider a small circle $C_{1}$ in the base sphere $S_{a}$. Roll along $C_{1}$, without slip nor twist, a ball $S_{b}$ (for concreteness, we do it externally), until it comes back to the initial point in $C_{1}$. The curvature is the following limit:

$\lim$ ( how much a frame attached to $S_{b}$ has rotated ) / ( area of the cap in $\left.S_{a}\right) \quad$, as $S_{a}$ shrinks .

Now, Proposition 2 tells that the corresponding curves have the same geodesic curvatures, so the contact point on $S_{b}$ must move in another circle $C_{2}$. After one turn arond $C_{1}$, the end point in $C_{2}$ is not the same as the initial point. For instance, the marker does several turns plus a fraction if $b$ is smaller than $a$. Moreover, proposition 1 allow us to replace the spheres by their enveloping cones.

\footnotetext{
${ }^{15}$ Cone rolling has many applications, ranging from transmission gears to leisure stuff, see http://www.kurt.com/gear.html, (no relation with one of the authors), http://www.1percent.com/store/cart/RLZENCONE.html (no relation with any of the authors, and http://kmoddl.library.cornell.edu/model.php?m=reuleaux (yes, we enjoy anything related to Cartan's 1:3 rolling!
} 
The equivalent problem is the following: let a cone $\Sigma_{2}$ roll around another cone $\Sigma_{1}$ until its generator returns to the same place. How much the position of a marker point $Q_{2}$ in $\Sigma_{2}$ has rotated around axis $\ell_{2}$ ? We call this angle divided by $2 \pi$ the holonomy. The reader should be able to sketch a figure. The moving cone $\Sigma_{2}$ rolls around the fixed cone $\Sigma_{1}$, having a common a different generatrix $g$ at every instant. Draw right triangles $V O_{1} q$ and $V O_{2} q$ where $V$ is the common vertex, $O_{i}$ in the axis of $\Sigma_{i}$ are the centers of the circles $C_{i}$, and $q$ is the contact point. Let $\alpha=\angle O_{1} V q$ and $\beta=\angle O_{2} V q$ the cones apertures, $\theta=\pi / 2-\alpha=\angle O_{1} q V, \phi=\pi / 2-\beta=\angle O_{q} q V$.

The answer is easy:

$$
\mid \text { holonomy } \mid=\text { length of the base circle } C_{1} / \text { length of } C_{2}=\sin (\alpha) / \sin (\beta)=\cos (\theta) / \cos (\phi) .
$$

Now by elementary trigonometry,

$$
a \tan (\theta)=b \tan (\phi)(=\ell) .
$$

which by the way says that the geodesic curvatures are

$$
\kappa_{C_{1}}=\kappa_{C_{2}}=1 / \ell .
$$

Thus

Moreover, from (3.12) we can solve for

$$
\text { holonomy }=-\frac{a}{b} \frac{\sin (\theta)}{\sin (\phi)}
$$

$$
\sin (\phi)=\frac{a}{b} \frac{\tan (\theta)}{\sqrt{1+\frac{a^{2}}{b^{2}} \tan ^{2}(\theta)}}
$$

so that

$$
\text { holonomy }=-\cos (\theta) \sqrt{1+\frac{a^{2}}{b^{2}} \tan ^{2}(\theta)} .
$$

Why the minus sign? The angle between the normals is $\alpha+\beta$, and since $\theta+\alpha=\pi / 2, \phi+\beta=\pi / 2$, both $\alpha$ and $\beta$ tend to $\pi / 2$ when $\theta$ (and so $\phi$ ) tends to zero. So the two normals tend to become opposite to each other ! Actually, here is one more twist: a frame attached to the sphere $S_{b}$ rotates by $2 \pi-2 \pi \mid$ holonomy|. The extra $2 \pi$ is due to the "Phileas Fogg effect" (known to mathematicians as Hopf's umlaufsatz). Now, the area of the cap in sphere $S_{a}$ is $2 \pi a^{2}(1-\cos (\theta)) \sim \pi a^{2} \theta^{2}$.

In short, we end up needing to compute the limit

$$
\lim _{\theta \rightarrow 0} \frac{2}{a^{2}} \frac{1-\sqrt{1+\frac{a^{2}}{b^{2}} \tan ^{2}(\theta)} \cos (\theta)}{\theta^{2}}
$$

This is a nice calculus exercise, the result being that the curvature is inded $\frac{1}{a^{2}}-\frac{1}{b^{2}}$ times the infinitesimal rotation about the normal around the contact point $q$ in sphere $S_{a}$.

Remark 7 Among the ratios $a / b$, two are exceptional: $a=b$ provides a holonomic distribution since the curvature vanishes. Cartan has used the 3:1 ratio to construct his exceptional Lie group $G_{2}$, see [18] 7]. We hoped that the corresponding rolling problems would have some special features, but that was with no avail.

\subsection{Hamiltonization of the sphere-sphere system}

We will compute step by step the 2-form $\omega_{N H}=d p_{\gamma} d \gamma+(J, K)$, and the reduced energy function $H: T^{*} S^{2} \rightarrow \mathbf{R}$, to convey the algorithmical nature of the method.

- The momentum mapping is given by $J\left(m_{q}, r\right)=m+q \times r$, see equation 2.10).

- Pairing the momentum with the curvature (3.11) along the global section $q \in S^{2}(a) \mapsto(q, I), q=a \gamma$ we get

$$
(J, K)=\left(1-\frac{b^{2}}{a^{2}}\right)(\gamma, M) d \operatorname{area}_{S^{2}}, M=A \Omega
$$

In order to match the domains, we follow the "clockwise diagram" presented in [27]. This diagram is a consequence of a Hamiltonian counterpart of Lagrangian reduction [20] using quasi-coordinates [40].

- The Legendre transform $T Q \mapsto T^{*} Q$ induced by the Lagrangian $T=\frac{1}{2}\left[\mu\left(1+\frac{b}{a}\right)^{2}\left|\dot{q}_{1}\right|^{2}+(A \Omega, \Omega)\right]$, at the point $\left(\dot{q}_{1}, \dot{R}\right)_{\left(q_{1}, R\right)}=$ $(v, \Omega)_{(a \gamma, I)} \in T_{(a \gamma, I)} Q$ is $\left(P_{v}, P_{I}\right)_{\left(q_{1}, R\right)}$, given by

$$
P_{I}=M=A \Omega, P_{v}=r=\mu\left(1+\frac{b}{a}\right)^{2} v
$$


- The Legendre transform of the horizontal lift $\operatorname{Hor}(\dot{\gamma})=(v, \Omega)=\left(-b \dot{\gamma},\left(1+\frac{b}{a}\right) \dot{\gamma} \times \gamma\right)$ :

$$
m=\left(1+\frac{b}{a}\right) A(\dot{\gamma} \times \gamma), r=\mu(-b)\left(1+\frac{b}{a}\right)^{2} \dot{\gamma}
$$

The end result is

$$
(J, K)=\left(1-\frac{b^{2}}{a^{2}}\right)\left(1+\frac{b}{a}\right)\left(\gamma, A(\dot{\gamma} \times \gamma) d S^{2}=\left(1-\frac{b}{a}\right)\left(1+\frac{b}{a}\right)^{2}\left(\gamma, A(\dot{\gamma} \times \gamma) d S^{2} .\right.\right.
$$

- To obtain the compressed Lagrangian $T_{\text {red }}$, we compute the Lagrangian at $\operatorname{Hor}(\dot{\gamma})$ :

$$
T_{\text {red }}=\frac{1}{2}\left(1+\frac{b}{a}\right)^{2}\left[\mu b^{2} \dot{\gamma}^{2}+(A(\gamma \times \dot{\gamma}), \gamma \times \dot{\gamma})\right]
$$

- The Legendre transform in the base, $p_{\gamma}=\partial T_{r e d} / \partial \dot{\gamma}=G(\gamma) \cdot \dot{\gamma}$ :

$$
p_{\gamma}=G(\gamma) \cdot \dot{\gamma}=\left(1+\frac{b}{a}\right)^{2}\left[\mu b^{2} \dot{\gamma}+A(\gamma \times \dot{\gamma}) \times \gamma\right]
$$

- The determinant of $G(\gamma)$, restricted to $T_{\gamma} S^{2}$ is

$$
F(\gamma)=\left[\operatorname{det} L e g_{\text {comp }}\right]=\left(I_{1} I_{2} I_{3}\right)\left(1+\frac{b}{a}\right)^{2}\left(\left(A^{-1} \gamma, \gamma\right)+\mu b^{2}\left[\frac{\gamma_{2}^{2}+\gamma_{3}^{2}}{I_{2} I_{3}}+\frac{\gamma_{1}^{2}+\gamma_{3}^{2}}{I_{1} I_{3}}+\frac{\gamma_{1}^{2}+\gamma_{2}^{2}}{I_{1} I_{2}}\right]+\frac{\mu^{2} b^{4}}{I_{1} I_{2} I_{3}}\right)
$$

Only this last assertion represents a nontrivial calculation, but that was done in [27], for the limit $a \rightarrow \infty$ of the rubber ball rolling over a plane. In that paper, we announced ((3.38), Proposition 3.6 of [27]):

Proposition 9 For $a=\infty$ (rubber ball rolling over a plane)

$$
d\left(f\left(\omega_{\text {can }}^{T^{*} S^{2}}+(J, K)\right)=0, f=F^{-1 / 2} .\right.
$$

Proof. This was checked directly by computer algebra, using spherical coordinates. It was useful to pull back the canonical two form in $T^{*} S^{2}$ to $T S^{2}$ via $p_{\gamma}=G(\gamma) \cdot \dot{\gamma}$. We can provide a Mathematica notebook for those interested.

We now show how to adapt this result for $b / a \neq 0$. First note that the conformality condition can we rewriten, when the base space of the bundle has dimension two, as

$$
d(\log f) \wedge \omega_{\text {can }}+d(J, K)=0 .
$$

From 3.16 and 3.18 we observe that $\omega_{\text {can }}$, when pulled back to $T S^{2}$, picks the factor $(1+b / a)^{2}$, whereas $(J, K)$ picks $(1-b / a)(1+b / a)^{2}$, that is, one fator $1-b / a$ surplus. Thus we can't have the same $f$ as in the case $a=\infty$, but if we change $f$ to $f^{1-b / a}$, that should work.

Theorem 4 The reduced rubber ball rolling over a ball is conformally symplectic,

$$
d\left[f_{a, b}(\gamma)\left(\omega_{c a n}^{T^{*} S^{2}}+(J, K)\right)\right]=0
$$

with conformal factor

$$
f_{a, b}(\gamma)=F^{\frac{b-a}{2 a}}
$$

Proof. For the suspicious reader, we can provide a Mathematica notebook using spherical coordinates. But a derivation (done by hand) follows from sphero-conical coordinates, as we show in the next section.

Remark 8 The presence of the exponent $\frac{b-a}{2 a}$ is somewhat mysterious, but it makes sense: when $b=a$, then $f \equiv 1$ (as it should be) since in that case $(J, K) \equiv 0$ and the problem becomes Hamiltonian. The predicted exponent from the results by Veselov and Veselova [61] or Fedorov and Jovanovic [31] would be $-1 / 2$, but this corresponds only to b/a $\rightarrow 0$. We believe that the problem resides on the singularity at the origin $a=0$, and the mystery will be resolved with a thorough study of bundles (3.7).

All these problems have symplectic forms parametrized by $(a, b)$ and the same Lagrangian on $T S^{2}$ containing two terms, of the form $\dot{\gamma}^{2}$ and $(A(\gamma \times \dot{\gamma}), \gamma \times \dot{\gamma})$, namely

$$
2 T /(1+b / a)^{2}=\mu b^{2} \dot{\gamma}^{2}+(A(\gamma \times \dot{\gamma}), \gamma \times \dot{\gamma})
$$

For $a / b=1$ (the case of equal spheres) we have $f \equiv 1$ and $(J, K)=0$, so we have the standard symplectic form in the base $T^{*} S^{2}$. Based on the classic integration by Jacobi of the ellipsoid geodesics problem, we could hope that the Hamiltonian separates in sphero-conical coordinates $\lambda_{1}, \lambda_{2}$, so the Hamiton-Jacobi method would work. We were be lead to a disgusting failure. 


\subsection{Sphero-conical coordinates}

This section is also standard material for the experts. Sphero-conical coordinates appear naturally if one wants to express (3.22) in the form $2 T=c_{1} \dot{\lambda}_{1}^{2}+c_{2} \dot{\lambda}_{2}^{2}$. To achieve this aim we need to diagonalize the positive quadratic form $(A x, x), x \in \mathbf{R}^{3}, A=$ $\operatorname{diag}\left(I_{1}, I_{2}, I_{3}\right)$ when restricted to subspaces defined by $(x, \gamma)=0$. This is a well known problem, going back to Lord Rayleigh. It boils down to extremize $(A x, x)$ subject to $\sum x_{j}^{2}=1$ and $(x, \gamma)=0$. Let us apply the Lagrange multiplier method, with an ammended objective function

$$
U=\frac{1}{2} \sum I_{j} x_{j}^{2}-\lambda\left(\sum x_{j}^{2}-1\right)-\mu \sum x_{j} \gamma_{j}
$$

The first order condition $\nabla U_{x}=0$ yields

$$
x_{j}=\mu \frac{\gamma_{i}}{I_{j}-\lambda}, 1 \leq j \leq 3 .
$$

Inserting in the constraints $\sum x_{i}^{2}=1, \sum x_{i} \gamma_{i}=0$ we get

$$
\begin{gathered}
\sum \frac{\gamma_{j}^{2}}{I_{j}-\lambda}=0 \\
\mu^{2}=1 /\left[\sum \frac{\gamma_{j}^{2}}{\left(I_{j}-\lambda\right)^{2}}\right] .
\end{gathered}
$$

This holds in $n$ dimensions, but let us keep working with $n=3$ for concreteness. If we order the parameters as $I_{1}<I_{2}<I_{3}$, equation 3.25 has two roots,

$$
\begin{aligned}
& I_{1}<\lambda_{1}=\lambda_{1}\left(\gamma ; I_{1}, I_{2}, I_{3}\right)<I_{2} \\
& I_{2}<\lambda_{2}=\lambda_{2}\left(\gamma ; I_{1}, I_{2}, I_{3}\right)<I_{3}
\end{aligned}
$$

(they can be found explicitly as the roots of a second degree equation in $\lambda$ thinking of the $\gamma_{j}$ as parameters).

For each choice of $\lambda$, 3.25 represents an elliptical cone in the three dimensional space $\left(\gamma_{1}, \gamma_{2}, \gamma_{3}\right)$. It is easy to see that the curves of intersection of these cones with the sphere $(\gamma, \gamma)=1 \mathrm{form}$ an orthogonal system of coordinates. This is because we know that the two vectors 3.24 that solve (3.23) are perpendicular, and moreover, these vectors are manifestly parallel to the gradients of 3.25 ). Thus we can anticipate that

$$
(A(\gamma \times \dot{\gamma}), \gamma \times \dot{\gamma})=b_{1} \dot{\lambda}_{1}^{2}+b_{2} \dot{\lambda}_{2}^{2}
$$

In order to find these coefficients $b_{1}, b_{2}$, we need to express $\left(\gamma_{1}, \gamma_{2}, \gamma_{3}\right)$ in terms of $\lambda_{1}, \lambda_{2}$. This is simply a matter of solving the linear system

with

$$
A\left(\gamma_{1}^{2}, \gamma_{1}^{2}, \gamma_{1}^{2}\right)^{t}=(1,0,0)^{t}
$$

$$
A=\left(\begin{array}{ccc}
1 & 1 & 1 \\
\left(I_{1}-\lambda_{I}\right)^{-1} & \left(I_{2}-\lambda_{I}\right)^{-1} & \left(I_{3}-\lambda_{I}\right)^{-1} \\
\left(I_{1}-\lambda_{2}\right)^{-1} & \left(I_{2}-\lambda_{2}\right)^{-1} & \left(I_{3}-\lambda_{2}\right)^{-1}
\end{array}\right)
$$

Inverting $A$ gives the desired explicit expression,

$$
\left(\gamma_{1}^{2}, \gamma_{2}^{2}, \gamma_{3}^{2}\right)=\left(\frac{\left(I_{1}-\lambda_{1}\right)\left(I_{1}-\lambda_{2}\right)}{\left(I_{1}-I_{3}\right)\left(I_{1}-I_{2}\right)}, \frac{\left(I_{2}-\lambda_{1}\right)\left(I_{2}-\lambda_{2}\right)}{\left(I_{2}-I_{3}\right)\left(I_{2}-I_{1}\right)}, \frac{\left(I_{3}-\lambda_{1}\right)\left(I_{3}-\lambda_{2}\right)}{\left(I_{3}-I_{1}\right)\left(I_{3}-I_{2}\right)}\right) .
$$

It follows that

and

$$
\frac{\partial \gamma}{\partial \lambda_{i}}=\frac{1}{2}\left(\frac{\gamma_{1}}{\lambda_{i}-I_{1}}, \frac{\gamma_{2}}{\lambda_{i}-I_{2}}, \frac{\gamma_{3}}{\lambda_{i}-I_{3}}\right), i=1,2
$$

$$
\gamma \times \frac{\partial \gamma}{\partial \lambda_{i}}=\frac{1}{2}\left(\frac{\gamma_{2} \gamma_{3}\left(I_{3}-I_{2}\right)}{\left(I_{2}-\lambda_{i}\right)\left(I_{3}-\lambda_{i}\right)}, \frac{\gamma_{1} \gamma_{3}\left(I_{1}-I_{3}\right)}{\left(I_{1}-\lambda_{i}\right)\left(I_{3}-\lambda_{i}\right)}, \frac{\gamma_{1} \gamma_{2}\left(I_{2}-I_{1}\right)}{\left(I_{1}-\lambda_{i}\right)\left(I_{2}-\lambda_{i}\right)}\right)
$$

The remaining calculations can be done by brute force, but it was less painful to use a computer algebra system. The results are:

Lemma 5

$$
\begin{gathered}
(A \dot{\gamma}, \dot{\gamma})=1 / 4 \frac{\left(\lambda_{2}-\lambda_{1}\right) \lambda_{1}}{\left(\lambda_{1}-I_{1}\right)\left(\lambda_{1}-I_{2}\right)\left(\lambda_{1}-I_{3}\right)} \dot{\lambda}_{1}^{2}+1 / 4 \frac{\left(\lambda_{1}-\lambda_{2}\right) \lambda_{2}}{\left(\lambda_{2}-I_{1}\right)\left(\lambda_{2}-I_{2}\right)\left(\lambda_{2}-I_{3}\right)} \dot{\lambda}_{2}^{2} \\
\|\dot{\gamma}\|^{2}=1 / 4 \frac{\lambda_{2}-\lambda_{1}}{\left(\lambda_{1}-I_{1}\right)\left(\lambda_{1}-I_{2}\right)\left(\lambda_{1}-I_{3}\right)} \dot{\lambda}_{1}^{2}+1 / 4 \frac{\lambda_{1}-\lambda_{2}}{\left(\lambda_{2}-I_{I}\right)\left(\lambda_{2}-I_{2}\right)\left(\lambda_{2}-I_{3}\right)} \dot{\lambda}_{2}^{2} \\
(A(\gamma \times \dot{\gamma}), \gamma \times \dot{\gamma})=b_{1} \dot{\lambda}_{1}^{2}+b_{2} \dot{\lambda}_{2}^{2}=\frac{1}{4} \frac{\lambda_{2}\left(\lambda_{2}-\lambda_{1}\right)}{\left(\lambda_{1}-I_{1}\right)\left(\lambda_{1}-I_{2}\right)\left(\lambda_{1}-I_{3}\right)} \dot{\lambda}_{1}^{2}+\frac{1}{4} \frac{\lambda_{1}\left(\lambda_{1}-\lambda_{2}\right)}{\left(\lambda_{2}-I_{1}\right)\left(\lambda_{2}-I_{2}\right)\left(\lambda_{2}-I_{3}\right)} \dot{\lambda}_{2}^{2} .
\end{gathered}
$$


Equation (3.31) indeed tell us that the ellipsoid geodesics problem separates (the standard metric $\dot{x}^{2}$ in the ellipsoid $\sum x_{j}^{2} / I_{j}=1$ is equivalent to the deformed metric $(A \dot{\gamma}, \dot{\gamma})$ in the sphere $\left.\sum \dot{\gamma}_{j}^{2}=1, \gamma_{j}=x_{j} / \sqrt{I_{j}}\right)$. The standard metric (3.32) on the sphere, which gives a superintegrable system, also appears separable in conical coordinates (but in a somewhat cumbersome format).

Let us now write the symplectic form 3.21 using $\lambda_{1}, \lambda_{2}, \dot{\lambda}_{1}, \dot{\lambda}_{2}$ as coordinates. This requires expressing the following objects in terms of those:

\section{Lemma 6}

$$
\begin{gathered}
d S^{2}=\left(\gamma, \frac{\partial \gamma}{\partial \lambda_{1}} \times \frac{\partial \gamma}{\partial \lambda_{2}}\right) d \lambda_{1} \wedge d \lambda_{2}=\frac{\lambda_{2}-\lambda_{1}}{\sqrt{\left(\lambda_{1}-I_{1}\right)\left(I_{2}-\lambda_{1}\right)\left(I_{2}-\lambda_{1}\right)\left(\lambda_{2}-I_{1}\right)\left(\lambda_{2}-I_{2}\right)\left(I_{3}-\lambda_{2}\right)}} d \lambda_{1} d \lambda_{2} \\
(\gamma, A(\dot{\gamma} \times \gamma))=\frac{1}{2} \gamma_{1} \gamma_{2} \gamma_{3}\left(\dot{\lambda}_{1}\left(\frac{I_{1}\left(I_{2}-I_{3}\right)}{\left(I_{2}-\lambda_{1}\right)\left(I_{3}-\lambda_{1}\right)}+\text { cyclic }\right)+\dot{\lambda}_{2}\left(\frac{I_{1}\left(I_{2}-I_{3}\right)}{\left(I_{2}-\lambda_{2}\right)\left(I_{3}-\lambda_{2}\right)}+\text { cyclic }\right)\right) \\
=\frac{1}{2} \sqrt{\frac{\left(\lambda_{2}-I_{1}\right)\left(\lambda_{2}-I_{2}\right)\left(I_{3}-\lambda_{2}\right)}{\left(\lambda_{1}-I_{1}\right)\left(I_{2}-\lambda_{1}\right)\left(I_{3}-\lambda_{1}\right)}} \dot{\lambda}_{1}+\frac{1}{2} \sqrt{\frac{\left(\lambda_{1}-I_{1}\right)\left(I_{2}-\lambda_{1}\right)\left(I_{3}-\lambda_{1}\right)}{\left(\lambda_{2}-I_{1}\right)\left(\lambda_{2}-I_{2}\right)\left(I_{3}-\lambda_{2}\right)}} \dot{\lambda}_{2} \\
\frac{\frac{\gamma_{2}^{2}}{I_{2}}+\frac{\gamma_{3}^{2}}{I_{3}}=\frac{\lambda_{1} \lambda_{2}}{I_{1} I_{2} I_{3}}}{I_{2}^{2}+\gamma_{3}^{2}}+\frac{\gamma_{1}^{2}+\gamma_{3}^{2}}{I_{1} I_{3}}+\frac{\gamma_{1}^{2}+\gamma_{2}^{2}}{I_{1} I_{2}}=\frac{\lambda_{1}+\lambda_{2}}{I_{1} I_{2} I_{3}} \\
T, K)=\frac{1}{2}\left(1-\frac{b}{a}\right)\left(1+\frac{b}{a}\right)^{2}\left(\lambda_{2}-\lambda_{1}\right)\left(\frac{\dot{\lambda}_{1}}{\left(\lambda_{1}-I_{1}\right)\left(\lambda_{1}-I_{2}\right)\left(\lambda_{1}-I_{3}\right)}+\frac{\lambda_{1}+\mu b^{2}}{\left(\lambda_{2}-I_{1}\right)\left(\lambda_{2}-I_{2}\right)\left(I_{3}-\lambda_{2}\right)}\right) \\
F(\gamma)=\left(1+\frac{b}{a}\right)^{2}\left(\lambda_{1}+\mu b^{2}\right)\left(\lambda_{2}+\mu b^{2}\right) \\
\frac{1}{8}\left(1+\frac{b}{a}\right)^{2}\left(\lambda_{2}-\lambda_{1}\right)\left(\frac{\lambda_{2}+\mu b^{2}}{\left(\lambda_{1}-I_{1}\right)\left(I_{2}-\lambda_{1}\right)\left(I_{3}-\lambda_{1}\right)} \dot{\lambda}_{1}^{2}+\frac{\lambda_{2}^{2}}{\left(\lambda_{2}-I_{1}\right)\left(\lambda_{2}-I_{2}\right)\left(I_{3}-\lambda_{2}\right)}\right) \\
p_{1}=\partial T / \partial \dot{\lambda}_{1}=\frac{1}{4}\left(1+\frac{b}{a}\right)^{2}\left(\lambda_{2}-\lambda_{1}\right) \frac{\lambda_{2}+\mu b^{2}}{\left(\lambda_{1}-I_{1}\right)\left(I_{2}-\lambda_{1}\right)\left(I_{3}-\lambda_{1}\right)} \dot{\lambda}_{1} \\
p_{2}=\partial T / \partial \dot{\lambda}_{2}=\frac{1}{4}\left(1+\frac{b}{a}\right)^{2}\left(\lambda_{2}-\lambda_{1}\right) \frac{\lambda_{1}+\mu b^{2}}{\left(\lambda_{2}-I_{1}\right)\left(\lambda_{2}-I_{2}\right)\left(I_{3}-\lambda_{2}\right)} \dot{\lambda}_{2}
\end{gathered}
$$

Putting everything together, we get:

Proposition 10 Darboux coordinates in new time:

$$
\begin{aligned}
\omega_{\text {conformal }} & =\left[\left(\lambda_{1}+\mu b^{2}\right)\left(\lambda_{2}+\mu b^{2}\right)\right]^{\frac{b-a}{2 a}} \omega_{n h}= \\
& =\left[\left(\lambda_{1}+\mu b^{2}\right)\left(\lambda_{2}+\mu b^{2}\right)\right]^{\frac{b-a}{2 a}}\left(d p_{1} d \lambda_{1}+d p_{2} d \lambda_{2}+\frac{1}{2}(1-b / a)\left(\frac{p_{1}}{\lambda_{2}+\mu b^{2}}-\frac{p_{2}}{\lambda_{1}+\mu b^{2}}\right) d \lambda_{1} d \lambda_{2}\right) \\
& =d\left(P_{1} d \lambda_{1}+P_{2} d \lambda_{2}\right) \quad \text { Darboux! }
\end{aligned}
$$

Proposition 11 The Hamiltonian in spheroconical coordinates: in new time

$$
d \tau / d t=F(\gamma)^{\frac{b-a}{2 a}}, F(\gamma)=\left(1+\frac{b}{a}\right)^{2}\left(\lambda_{1}+\mu b^{2}\right)\left(\lambda_{2}+\mu b^{2}\right)
$$

defining

$$
P_{1}=\left[\left(\lambda_{1}+\mu b^{2}\right)\left(\lambda_{2}+\mu b^{2}\right)\right]^{\frac{b-a}{2 a}} p_{1}, P_{2}=\left[\left(\lambda_{1}+\mu b^{2}\right)\left(\lambda_{2}+\mu b^{2}\right)\right]^{\frac{b-a}{2 a}} p_{2} .
$$

the Hamiltonian becomes

$$
2 H=\frac{p_{1}^{2}}{c_{1}}+\frac{p_{2}^{2}}{c_{2}}=\frac{P_{1}^{2}}{\left[\left(\lambda_{1}+\mu b^{2}\right)\left(\lambda_{2}+\mu b^{2}\right)\right]^{\frac{b-a}{a}} c_{1}}+\frac{P_{2}^{2}}{\left[\left(\lambda_{1}+\mu b^{2}\right)\left(\lambda_{2}+\mu b^{2}\right)\right]^{\frac{b-a}{a}} c_{2}}
$$

with

$$
c_{1}=\frac{1}{4}\left(1+\frac{b}{a}\right)^{2}\left(\lambda_{2}-\lambda_{1}\right) \frac{\lambda_{2}+\mu b^{2}}{\left(\lambda_{1}-I_{1}\right)\left(I_{2}-\lambda_{1}\right)\left(I_{3}-\lambda_{1}\right)}, c_{2}=\frac{1}{4}\left(1+\frac{b}{a}\right)^{2}\left(\lambda_{2}-\lambda_{1}\right) \frac{\lambda_{1}+\mu b^{2}}{\left(\lambda_{2}-I_{1}\right)\left(\lambda_{2}-I_{2}\right)\left(I_{3}-\lambda_{2}\right)} .
$$




\section{Some directions for research}

\subsection{Nonintegrability.}

If the rolling ball has its mass concentrated at the geometric center, then the inertias $I_{j}$ vanish. Hence, the problem with $I_{j}=\varepsilon \bar{I}_{j}$, is a perturbation of the geodesics on the sphere. As a possible attempt to prove nonintegrability rigorously is to compute the monodromy of the linearized equations along a great circle (solution of the unperturbed problem) and apply Melnikov or Moralis-Ramis methods. Parhaps this may be done directly in $\gamma \in \mathbf{R}^{3}$. In sphero-conical coordinates, using rescaled variables $\lambda_{j}=\varepsilon \bar{\lambda}_{j}$ and new time with factor

$$
F(\gamma)=\left(1+\frac{b}{a}\right)^{2}\left(\varepsilon \lambda_{1}+\mu b^{2}\right)\left(\varepsilon \lambda_{2}+\mu b^{2}\right)
$$

we obtain (dropping the bars) the following Hamiltonian perturbation of the geodesics on the sphere:

$$
2 H=\frac{P_{1}^{2}}{\left[\left(\varepsilon \lambda_{1}+\mu b^{2}\right)\left(\varepsilon \lambda_{2}+\mu b^{2}\right)\right]^{\frac{b-a}{a}} a_{1}}+\frac{P_{2}^{2}}{\left[\left(\varepsilon \lambda_{1}+\mu b^{2}\right)\left(\varepsilon \lambda_{2}+\mu b^{2}\right)\right]^{\frac{b-a}{a}} a_{2}}
$$

with

$$
a_{1}=\frac{1}{4}\left(1+\frac{b}{a}\right)^{2}\left(\lambda_{2}-\lambda_{1}\right) \frac{\varepsilon \lambda_{2}+\mu b^{2}}{\left(\lambda_{1}-I_{1}\right)\left(I_{2}-\lambda_{1}\right)\left(I_{3}-\lambda_{1}\right)}, a_{2}=\frac{1}{4}\left(1+\frac{b}{a}\right)^{2}\left(\lambda_{2}-\lambda_{1}\right) \frac{\varepsilon \lambda_{1}+\mu b^{2}}{\left(\lambda_{2}-I_{1}\right)\left(\lambda_{2}-I_{2}\right)\left(I_{3}-\lambda_{2}\right)} .
$$

\subsection{Rolling with sliping but no twisting and Rattleback phenomena}

From the mathematical point of view, it is legitimate to study systems subject to the no-twist constraint, sliding allowed. The question is whether these constraints could be (even approximately) realized in practice. Nonholonomic constraints are approximately realized as limits of certain collisional systems. It would be interesting to conceive a "peg-leg" version for no-sliping and the no-twist constraints 16 .

We believe that the dynamical torque 2.25) the heart of the rattleback rotation reversal phenomenon. There is a extensive literature on the topic, but we would like to see an explanation based on the joint geometries of $\Sigma_{2}$, say with $\Sigma_{1}$ being the plane. Is there a rubber rattleback? Here the idea is not looking at rotations (which are forbidden) but at translation reversal.

\subsection{Skiding dynamics: mathematical hockey}

As far as we know holonomic systems $T\left(q_{1}, R, \dot{q}_{1}, \dot{R}\right)$ given by (2.4), describing the motion of a body $\mathcal{B}$ skiding over a surface $\Sigma_{1}$ have not been studied systematically. In general the rotational and surface dynamics should be coupled and some interesting effects of mathematical hockey may be anticipated 17 . A first project in this direction is to explore symmetries (as we discussed briefly).

\subsection{Rubber rolling of surfaces of revolution over a sphere}

Conditions under which a generalized Chaplygin system has an invariant measure are given in [17]. It would be interesting to make explicit which condition results for marble rolling of an arbitrary surface $\Sigma_{2}$ over a sphere or a plane. Also, implementating proposition 3 on exemples such as bodies of revolution and ellipsoids is in order, as well as the higher dimensional extensions [30, 36]. These situations have been studied in the marble rolling context, see tables 1 and 2 of [10]. On another talk, rubber rolling of an homogenous sphere over arbitrary surfaces $\Sigma_{1}$, see [11] for the marble counterpart.

\subsection{Suslov rolling}

While the usual no-slip constraint is enforced, the no-twist constraint $\omega_{3}=0$ is replaced by $\Omega_{3}=0$, that is, one imposes that the third component of angular velocity in the body frame should vanish. This constraint can be implemented by a inner spherical support.

\subsection{Rubber rolling and Yang-Mills}

Among all convex bodies $\mathcal{B}$ with the same area, rolling over a sphere, which one minimizes the Yang-Mills functional? This functional, important for gauge theories, is given by

$$
\int_{S^{2}}\|K\|^{2} d A
$$

${ }^{16}$ We thank Andy Ruina for a discussion on this subject; see http://ruina.tam.cornell.edu/

${ }^{17}$ Hockey (see http://www.exploratorium.edu/hockey/) could be even more exciting with a strictly convex puck. 
where $K$ is the curvature of the connection. Here we restric ourselves to rubber connections $\phi_{\mathcal{B}}$ on the bundle $S O(3) \times S^{2}$. A nice exercise is to compute the rubber connection 1-form and its curvature, for a tri-axial ellipsoid rolling over a sphere.

A reasonable conjecture is that the sphere minimizes the YM functional. Clearly, the minimum attains the value zero when the area of B is the same as the area of $S_{a}$.

Yang Mills connections over Riemann surfaces were considered on an important paper by Atiyah and Bott [3]. Particularize to rolling problems when the base is a surface of constant negative curvature.

Acknowledgements. Both authors thank Prof. Mikhail A. Sokolovskiy for inviting us to the IUTAM symposium 2006 on Hamiltonian Dynamics, Vortex Structures, Turbulence, at the Steklov Institute, Moscow, http://conf2006.rcd.ru/ where me met Alexei Borisov, Ivan Mamaev, Alexander Kilin, Yuri Fedorov, and renewed contact with Anatoly Neishtadt. This meeting was a wonderful experience. JK slso thanks Richard Murray and Jerry Marsden for hosting a Fulbright visit, Winter 2005, where this research started: a great time in company of Tudor Ratiu, Marco Castrillon, and Hernan Cendra.

\section{References}

[1] Arnold, V. I., Mathematical Methods of Classical Mechanics, volume 60 of Graduate Texts in Math. Springer-Verlag, First Edition 1978, Second Edition, 1989.

[2] Arnold, V. I., Kozlov, V.V., A. I. Neishtadt, A.I., Mathematical aspects of classical and celestial mechanics, in Arnold, V. I., editor, Dynamical Systems III, Encyclopaedia of Mat. Sciences, Springer-Verlag, 1988.

[3] Atiyah, M., Bott, R., The Yang-Mills Equations over Riemann Surfaces, Philosophical Transactions of the Royal Society of London. Series A, Mathematical and Physical Sciences, 308:1505, 1983.

[4] Blackall, C.J., On volume integral invariants of non-holonomic dynamical systems, Amer. J. Math. 63:1, 155-168, 1941.

[5] Bloch, A.M., Nonholonomic mechanics and control, Springer-Verlag, 2003.

[6] Bloch, A.M., Krishnaprasad, P.S., Marsden, J.E., Murray, R.M., Nonholonomic mechanical systems with symmetry, Arch. Ratl. Mech. Anal., 136, 21-99.1996.

[7] Bor, G., and Montgomery, R., $G_{2}$ and the "rolling distribution", preprint.

[8] Borisov, A. V., Mamaev, I.S., Chaplygin's ball rolling problem is Hamiltonian, Mathematical Notes (Matematicheskie Zametki), 70:5, 793-795, 2001.

[9] Borisov, A. V., Mamaev, I.S., Obstacle to the Reduction of Nonholonomic Systems to the Hamiltonian Form, Doklady Physics USSR, 47:12, 892-894, 2002.

[10] Borisov, A. V., Mamaev, I. S., The Rolling Body Motion Of a Rigid Body on a Plane and a Sphere. Hierarchy of Dynamics, Regular and Chaotic Dyn., 7:2, 177-200, 2002.

[11] Borisov A.V., Mamaev I.S. and Kilin A.A., The rolling motion of a ball on a surface. New integrals and hierarchy of dynamics, Regular and Chaotic Dyn., 7:2, 201-218, 2002.

[12] Borisov, A.V., Mamaev, I.S., Isomorphism and Hamilton representation of some nonholonomic systems, em Siberian Mathematical Journal, 48:11,2007, to appear. see preliminary version at arXiv: nlin.SI/0509036 v1 (Sept. 21 2005).

[13] Borisov, A.V, Mamev, I.S., this issue.

[14] Bryant, R., Hsu,L., Rigidity of integral curves of rank 2 distributions, Inventiones Mathematicae 114, 435-461, 1993.

[15] Cantrijn, F., de Léon, M., Marrero, J.C., de Diego, D., Reduction of nonholonomic mechanical systems with symmetries, Rep. Math. Phys. 42:1/2, 25-45, 1998.

[16] Cantrijn, F., de Léon, M., de Diego, D., On almost-Poisson structures in nonholonomic mechanics, Nonlinearity 12 , 721-737, 1999.

[17] Cantrijn, F., Cortés, J., de Léon, M., de Diego, D., On the geometry of generalized Chaplygin systems, Math. Proc. Camb. Phil. Soc. 132, 323-351, 2002.

[18] Cartan, É., Le systèmes de Pfaff à cinq variables et les équations aux dèrivèes partielles du second ordre, Ann. Sci. Ècole Normale 27:(3), 109-192, 1910.

[19] Cartan, É., Sur la represéntation géométrique des systèmes matériels non holonomes, Proc. Int. Congr. Math. Bologna 4, 253-261, 1928.

[20] Cendra, H., Marsden, J. E., Ratiu, T. S., Geometric mechanics, Lagrangian reduction and nonholonomic systems, Mathematics Unlimited-2001 and Beyond, (B. Enguist and W. Schmid, eds.), Springer-Verlag, New York, 221-273, 2001. 
[21] Chaplygin, S.A., On the theory of the motion of nonholonomic systems. Theorem on the reducing factor, Mat. Sbornik, 28, 303-314, 1911.

[22] Chaplygin, S.A., On a ball's rolling on a horizontal plane, Reg. Chaot. Dyn., 7:2, 131-148, 2002. Original paper in Math. Sbornik 24, 139-168, 1903.

[23] Cortés, J., Geometric, Control and Numerical Aspects of Nonholonomic Systems, Springer-Verlag, 2002.

[24] Cortés, J., de Léon, M., de Diego, D., Martínez, S., Geometric description of vakonomic and nonholonomic dynamics; comparison of solutions, SIAM J. Control Optim. 41:5, 1389-1412, 2003.

[25] Cushman, R., Bates, L., Global aspects of Classical Integrable Systems, Birkhäuser, Basel, 1997.

[26] Duistermaat, J.J. [2000], Chapygin's sphere, in R. Cushman, J. J. Duistermaat and J. Śniatycki: Chaplygin and the Geometry of Nonholonomically Constrained Systems, 2000 (in preparation). See arxiv:

[27] Ehlers, K., Koiller, J., Montgomery, R., Rios, P.M., Nonholonomic systems via moving frames: Cartan equivalence and Chaplygin hamiltonization, In The Breadth of Symplectic and Poisson Geometry, Festschrift in Honor of Alan Weinstein, ed. by J.Marsden and T.Ratiu, Birkhuser, Boston, 2005

[28] Ehlers, K., Koiller, J., Rubber rolling: geometry and dynamics of 2-3-5 distributions, in Proceedings IUTAM symposium 2006 on Hamiltonian Dynamics, Vortex Structures, Turbulence, at the Steklov Institute, Moscow, to appear.

[29] Ehlers, K., Koiller, J., Classification of the 2-3-5 nonholonomic geometries, in preparation.

[30] Fedorov, Yu. N., Kozlov, V.V., Various aspects of n-dimensional rigid body dynamics, in Kozlov, V.V. (editor) Dynamical Systems in Classical Mechanics, volume 168 of AMS Translations series 2, 1995.

[31] Fedorov, Yu. N., Jovanovic, B., Nonholonomic LR systems as Generalized Chaplygin systems with an Invariant Measure and Geodesic Flows on Homogeneous Spaces, J. Nonlinear Science, 14:1, 341-381, 2004 ( arxiv.org/abs/math$\mathrm{ph} / 0307016,2003)$.

[32] Haller, S., Rybicki, T., On the group of diffeomorphisms preserving a locally conformal symplectic structure, Ann. Global Anal. Geom. 17, 475-502, 1999.

[33] Haller, S., Rybicki, T., Symplectic reduction for locally conformal symplectic manifolds, J. Geom. Phys. , 37, 262-271, 2001.

[34] Hertz, H., The principles of mechanics presented in a new form by Heinrich Hertz, with an introduction by H. von Helmholtz, Macmillan, London, New York, 1899.

[35] Iliyev, Il., On the conditions for the existence of the reducing Chaplygin factor, P.M.M. USSR 49:2, 295-301, 1985.

[36] Jovanovic, B. , Some multidimensional integrable cases of nonholonomic rigid body dynamics, Regular \& Chaotic Dynamics 8:1, 125-132, 2003.

[37] Kilin, A.A., The Dynamics of Chaplygin ball: the qualitative and computer analysis,Reg. Chaotic Dynamics 6:3, 291-306, 2001.

[38] Kobayashi, M.H., Oliva, W.M., A note on the conservation of energy and volume in the setting of nonholonomic mechanical systems, Qual. Theory Dyn. Systems, 5, 255-283, 2004.

[39] Koiller, J., Reduction of some classical non-holonomic systems with symmetry, Arch. Rational Mech. Anal. 118, 113-148, 1992.

[40] Koiller, J., Rios, P.M., Ehlers, K., Moving frames for cotangent bundles, Rep. Math. Phys., 49:2/3, 225-238, 2002.

[41] Kozlov, V.V., On the integration theory of equations of nonholonomic mechanics, Reg. Chaot. Dyn., 7:2, 161-176, 2002.

[42] Koon, W. S., Marsden, J.E., The Hamiltonian and Lagrangian approaches to the dynamics of nonholonomic systems, Rep. Math. Phys. , 40, 21-62, 1997.

[43] Koon, W. S., Marsden, J.E., Poisson reduction for nonholonomic mechanical systems with symmetry, Reports on Math. Phys., 42, 101-134, 1998.

[44] Kupka, I., Oliva, W.M., The Non-Holonomic Mechanics, J. Diff. Equations 169, 169-189, 2001.

[45] Levi, M.[1996], Composition of rotations and parallel transport, Nonlinearity 9, 413-419, 1996.

[46] Marle, C.M., Various approaches to conservative and nonconservative nonholonomic systems, Rep. Math. Phys. 42:1/2, 211-229, 1998.

[47] Marle, C.M., On symmetries and constants of motion in Hamiltonian systems with nonholonomic constraints, Banach Center Publ., 59, 223-242, 2003.

[48] Marle, C.M., From momentum maps and dual pairs to symplectic and Poisson groupoids, In: The Breadth of Symplectic and Poisson Geometry, Festschrift in Honor of Alan Weinstein, Birkhauser, Boston, ed. by J. Marsden and T. Ratiu, 493-523, 2005. 
[49] Marsden, J.E., and Ratiu, T., Introduction to Mechanics and Symmetry, Texts in Applied Mathematics vol. 17, 2nd ed. , Springer, New York, 1999.

[50] Marsden, J.E., Weinstein, A., Reduction of symplectic manifolds with symmetry, Rep. Math. Phys. 5, 121-130, 1974.

[51] Montgomery, R., A Tour of Subriemannian Geometries, their Geodesics, and Applications, AMS Surveys and Monographs 91, 2002

[52] Naranjo, L.G., Ph.D. thesis, University of Arizona (see http://math.arizona.edu/ luisg/).

[53] Neimark, J.I., N. A. Fufaev, N.A., Dynamics of nonholonomic systems volume 33 of AMS Translations of Mathematical Monographs, Providence, 1972.

[54] Oliva, W.M., Geometric Mechanics, volume 1798 of Springer Lecture Notes in Mathematics Springer Verlag, 2002.

[55] van der Schaft, A.J., Port-Hamiltonian systems: an approach to modeling and control of complex physical systems, Proceedings of the Sixteenth International Symposium on Mathematical Theory of Networks and Systems (MTNS2004), Leuven, Belgium, July 5-9, 2004.

[56] Śniatycli, J., Nonholonomic Noether theorem and reduction of symmetries, Rep. Math. Phys. 42:1/2, 5-23, 1998.

[57] Stanchenko, S.V., Non-holonomic Chaplygin systems, P.M.M. USSR 53:1, 11-17, 1985.

[58] Struik, D. J., Lectures on classical differential geometry, Addison-Wesley, 1950.

[59] Vaisman, I., Locally conformal symplectic manifolds. Internat. J. Math. Math. Sci. 8:3, 521-536, 1985.

[60] Veselov, A. P., Veselova, L. E., Flows on Lie groups with a nonholonomic constraint and integrable non-Hamiltonian systems. (Russian), Funktsional. Anal. i Prilozhen. 20:4, 65-66. English translation: Functional Anal. Appl. 20:4,308309, 1986.

[61] Veselov, A. P., Veselova, L. E., Integrable nonholonomic systems on Lie groups. (Russian) Mat. Zametki 44:5 604-619, 701; translation in Math. Notes 44:5/6, 810-819, 1989.

[62] Yoshimura, H., Marsden, J., Dirac Structures and Implicit Lagrangian Systems in Electric Networks, 17th International Symposium on Mathematical Theory of Networks and Systems, Kyoto, 2006.

[63] Wade, A., Conformal Dirac structures, Lett. Math. Phys. 53, 331-348, 2000.

[64] Zenkov, D.V., Bloch, A.M., Invariant measures of nonholonomic flows with internal degrees of freedom, Nonlinearity 16, 1793-1807, 2003. 